\title{
Symmetries and Their Breaking in the Fundamental Laws of Physics
}

\author{
Jose Bernabeu \\ Department of Theoretical Physics, University of Valencia and IFIC, Joint Centre Univ. Valencia-CSIC, \\ E-46100 Burjassot, Spain; Jose.Bernabeu@uv.es
}

Received: 22 June 2020; Accepted: 29 July 2020; Published: 6 August 2020

\begin{abstract}
Symmetries in the Physical Laws of Nature lead to observable effects. Beyond the regularities and conserved magnitudes, the last few decades in particle physics have seen the identification of symmetries, and their well-defined breaking, as the guiding principle for the elementary constituents of matter and their interactions. Flavour SU(3) symmetry of hadrons led to the Quark Model and the antisymmetric requirement under exchange of identical fermions led to the colour degree of freedom. Colour became the generating charge for flavour-independent strong interactions of quarks and gluons in the exact colour SU(3) local gauge symmetry. Parity Violation in weak interactions led us to consider the chiral fields of fermions as the objects with definite transformation properties under the weak isospin $\mathrm{SU}(2)$ gauge group of the Unifying Electro-Weak $\mathrm{SU}(2) \times \mathrm{U}(1)$ symmetry, which predicted novel weak neutral current interactions. $\mathrm{CP}$-Violation led to three families of quarks opening the field of Flavour Physics. Time-reversal violation has recently been observed with entangled neutral mesons, compatible with CPT-invariance. The cancellation of gauge anomalies, which would invalidate the gauge symmetry of the quantum field theory, led to Quark-Lepton Symmetry. Neutrinos were postulated in order to save the conservation laws of energy and angular momentum in nuclear beta decay. After the ups and downs of their mass, neutrino oscillations were discovered in 1998, opening a new era about their origin of mass, mixing, discrete symmetries and the possibility of global lepton-number violation through Majorana mass terms and Leptogenesis as the source of the matter-antimatter asymmetry in the universe. The experimental discovery of quarks and leptons and the mediators of their interactions, with physical observables in spectacular agreement with this Standard Theory, is the triumph of Symmetries. The gauge symmetry is exact only when the particles are massless. One needs a subtle breaking of the symmetry, providing the origin of mass without affecting the excellent description of the interactions. This is the Brout-Englert-Higgs Mechanism, which produces the Higgs Boson as a remnant, discovered at CERN in 2012. Open present problems are addressed with by searching the New Physics Beyond-the-Standard-Model.
\end{abstract}

Keywords: flavour families; colour charges; gauge symmetries; chirality; discrete symmetries; neutrinos; spontaneous breaking

\section{Symmetry as Guiding Principle for Particles and Interactions}

In ordinary life, we observe symmetry of objects, like characteristic features of geometrical forms, material objects or biological bodies. The concept is related to the invariance of the object under definite transformations: One object is symmetric if, after a transformation is applied, the result remains the same, i.e., it remains "invariant". However, we also observe Symmetry Breaking, which is particularly of interest when it is not a random effect but follows a definite pattern. In Figure 1, we show the three-span arch of the FermiLab entrance, near Chicago, which appears perfectly symmetric when viewed from below, but has a calculated asymmetry from its other views. Symmetry and Symmetry 
Breaking are very important concepts in the field of elementary particle physics, however not referring to objects, but to the Fundamental Laws of Physics.

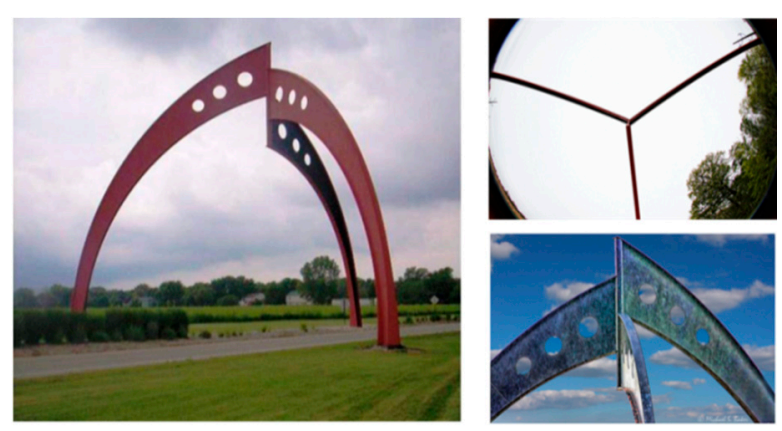

Figure 1. Symmetry Breaking.

We show here how Symmetry has acted as a guiding principle for both the existence of new particles and the formulation of interactions. One can claim that "Symmetry dictates Interaction", as stated by Yang. In Quantum Mechanics, the Symmetry is implemented by a Unitary Transformation $\hat{U}$ acting on states and observables. If the Dynamics, described by the Hamiltonian $\hat{H}$, are Invariant under the transformation, one can obtain

$$
[\hat{H}, \hat{U}]=0
$$

For continuous groups characterized by a parameter "a", the transformation $\hat{U}=\exp \{-i \hat{G} a / \hbar\}$ is generated by $\hat{G}=\hat{G}^{+}$and one obtains immediately

$$
\frac{d}{d t}\langle\hat{G}\rangle=i\langle[\hat{H}, \hat{G}]\rangle=0
$$

As $\hat{G}$ is Hermitian, it corresponds to an Observable that satisfies a Conservation Law if $\hat{U}$ is symmetry of $\hat{H}$. Well known examples are momentum for translations, angular momentum for rotations or charge for gauge symmetry. For local gauge symmetry, the requirement of Invariance leads to a Covariant Derivative with a Mediator Field responsible for interactions. This is valid for either QED with the Abelian U(1) gauge group or non-Abelian gauge groups with the interaction field transforming as the adjoint representation.

In Section 2, we develop the ideas leading from hadrons to quarks and the symmetries of strong interactions. In Section 3, a parallel discussion is made for electroweak interactions starting from Parity Violation and leading to the Standard Model with neutral currents and the need for charm, plus the third family of bottom and top quarks, including Quark-Lepton Symmetry. Section 4 discusses Discrete Symmetries, with the observation and implications of the independent breaking of CP and T, as well as tests of CPT. In Section 5, the fascinating history of neutrino physics is summarized and the present open questions of CP-Violation in the lepton sector and Global Lepton Number Violation are discussed. Section 6 presents the Brout-Englert-Higgs Mechanism for the Origin of Mass, breaking the ElectroWeak Gauge Symmetry. Some Conclusions and Outlook in the field of Symmetries are provided in Section 7.

\section{Quarks and Strong Interactions}

The proliferation of non-strange and strange Hadrons in the 1960's of the 20th century led to the Eightfold Way of Gell, Mann and Ne'eman with the use of the Flavour SU(3) symmetry. The fundamental representations $3, \overline{3}$ are the elementary building blocks for all higher-dimensional representations. In terms of their basis states, Mesons are $\mathrm{q}-\overline{\mathrm{q}}$ states $3 \times \overline{3}=1+8$, Baryons are $\mathrm{q}-\mathrm{q}-\mathrm{q}$ states $3 \times 3 \times 3=1+8 \mathrm{~s}+8_{\mathrm{a}}+10$, with three quark $\mathrm{q}=\mathrm{u}$, d, s states. In Figure 2, the octet and decuplet 
representations of Baryons are given in terms of third component of Isospin $\mathrm{I}_{3}$ and hypercharge $\mathrm{Y}$ axes. According to the Gell-Mann-Nishijima rule, the electric charge is $Q=I_{3}+Y / 2$, with $Y=B+S, B$ the baryonic number and $\mathrm{S}$ strangeness.

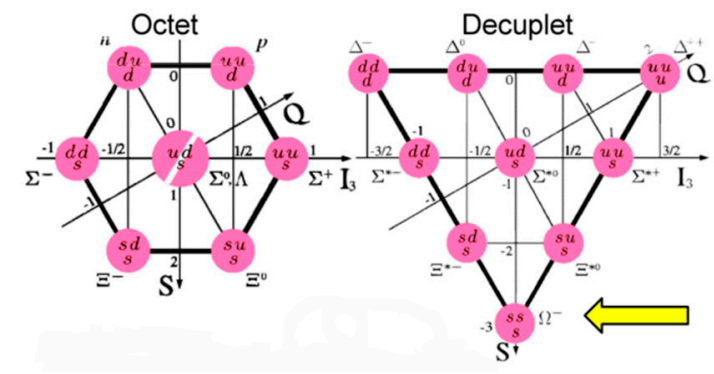

Figure 2. Octet and Decuplet of Baryons.

At the time of this formulation, the $\Omega^{-}$had not been detected. Its later discovery was a great triumph of the whole scheme.

\subsection{Are Quarks Real?}

For some time, however, the quark model for hadrons [1] was considered by the scientific community as a mere theoretical construct to describe the classification of hadrons in the SU(3) symmetry. The question was "Are Quarks real?" Since 1969, deep inelastic scattering experiments [2] at SLAC (the name of a Physics Centre) showed that the proton contained much smaller, point-like constituents and was therefore not an elementary particle. Physicists were reluctant to firmly identify these objects with quarks at the time, instead calling them "partons"—a term coined by Feynman. The partons that were observed at SLAC would later be identified as up and down quarks. Nevertheless, "parton" remains in use as a collective term for the constituents of hadrons (quarks, antiquarks and gluons). We do know at present that Leptons (electrons, muons, neutrinos) find partons in the proton with high momentum transfer events.

A "jet" is a narrow cone of hadrons produced by the hadronization of a parton. Jets were observed for the first time in the $\mathrm{e}^{+} \mathrm{e}^{-}$annihilation into hadrons at the SPEAR storage ring [3] and interpreted in terms of quarks. Quarks therefore exist, but they cannot propagate asymptotically. Quarks are therefore confined.

One of the reasons why the idea of real quarks was seen with skepticism was the problem of quarks with the exchange symmetry associated with the spin-statistics connection. It is easily realized with the $\Delta^{++}$puzzle: The state $\mathbf{u}^{\uparrow} \mathbf{u}^{\uparrow} \mathbf{u}^{\uparrow}$ with the third component of total spin $S_{3}=+3 / 2$ is evidently symmetric under exchange of flavour $(u)$, quark spin $\left(s_{3}=+1 / 2\right)$ and space $(L=0)$ degrees of freedom of the three quarks.

If quarks are real fermions and satisfy the exchange symmetry, a new degree of freedom is necessary for quarks, the "colour" ( $r, g, b)$ being antisymmetric for its exchange in baryons. Precisely the singlet colour wave function

$$
\Psi_{c}^{q q q}=\frac{1}{\sqrt{6}}(r g b-r b g+g b r-g r b+b r g-b g r)
$$

is antisymmetric, therefore qqq states exist, but these hadrons are colourless. We conclude that colour is confined, therefore colourful quarks are confined. For the requirement of antisymmetry, we need a number $\mathrm{N}_{\mathrm{c}}=3$ of colours. Experimental evidence indicates that $\mathrm{N}_{\mathrm{c}}=3$ came from the interpretation of $\mathrm{e}^{+} \mathrm{e}^{-} \rightarrow$ hadrons in terms of $q \bar{q}$ production, with a cross-section predicted to be proportional to $\mathrm{N}_{\mathrm{c}}$. 


\subsection{The Colour Charge}

The Colour Charge appears as Generator of an exact SU(3) ${ }_{c}$ Local Gauge Symmetry, leading to the Colour Interaction of Quarks in the Fundamental Representation, mediated by eight massless Gluons in the Adjoint Representation. This interaction is flavour-blind and only the quark mass terms break flavour independence. The origin of the quark mass terms should then be external to this Quantum Chromodynamics (QCD) theory. The field tensor is covariant $(\mathrm{A}=1, \ldots, 8)$ leads to the self-interaction of the vector gluon field $\mathrm{A}_{\mu}^{A}$ in the Lagrangian term $-\frac{1}{4} F_{\mu \nu}^{\mathrm{A}} F^{\mathrm{A} \mu \nu}$

$$
F_{\mu v}^{A}=\partial_{\mu} \mathrm{A}_{v}^{\mathrm{A}}-\partial_{v} \mathrm{~A}_{\mu}^{\mathrm{A}}-g_{s} f_{A B C} \mathrm{~A}_{\mu}^{\mathrm{B}} \mathrm{A}_{v}^{C}
$$

with $g_{s}$ the quark-gluon coupling and $f_{A B C}$ the structure constants of the $\mathrm{SU}(3)_{\mathrm{c}}$ group. All coloured objects have strong interaction with gluons, therefore quarks have strong interactions with gluons, and gluons with themselves. Gluons have colour, so they are confined like quarks. Gluon Jets were first observed in the annihilation $\mathrm{e}^{+} \mathrm{e}^{-} \rightarrow q \bar{q} g$ to three jets by the TASSO experiment [4] at the PETRA accelerator in the DESY laboratory.

The QCD coupling constant $\alpha_{S}=g_{S}{ }^{2} /(4 \pi)$ is dimensionless, therefore the classical field theory in the chiral (massless) limit is Scale Invariant. There is a Conformal Symmetry. However, in the perturbative treatment of the QCD quantum theory, predictions for observables are made in terms of the renormalized coupling $\alpha_{S}\left(\mu_{R}^{2}\right)$, which is a function of the renormalization scale. Taking it close to the momentum transfer $\mathrm{Q}^{2}, \alpha_{\mathrm{S}}\left(\mathrm{Q}^{2}\right)$ indicates the effective strength of the interaction.

\subsection{Asymptotic Freedom}

The coupling runs with the renormalization scale $\mu_{R}^{2}$ and this running coupling satisfies the Renormalization Group Equation controlled by the QCD $\beta\left(\alpha_{s}\right)$ function. The 1 loop $\beta$ function coefficient has contributions to the gluon self-energy from gluon self-couplings and fermion couplings with opposite signs. The dominance of the first term gives to QCD, distinct to QED, the property of Asymptotic Freedom [5,6]. The approximate analytic solution is

$$
\alpha_{s}\left(\mu_{R}^{2}\right)=\left(b_{0} \ln \left(\frac{\mu_{R}^{2}}{\Lambda^{2}}\right)\right)^{-1}, \quad b_{0}=\left(33-2 n_{f}\right) /(12 \pi)
$$

with $n_{f}$ as the number of flavour fermions and $\Lambda$ a constant of integration, representing the non-perturbative scale of $\mathrm{QCD}$. The running coupling has been experimentally demonstrated with $\Lambda \sim 250 \mathrm{MeV}$. The Dimensional Transmutation from $\alpha_{\mathrm{s}}$ to $\Lambda$ thus originates in the quantum Conformal Anomaly breaking the conformal symmetry. This $\Lambda$ is responsible of the nucleon mass and, as a consequence, the baryonic mass of the Universe.

\section{Chirality and Electroweak Interaction}

Parity Violation by Weak Interactions was postulated [7] in the 1950's of the 20th century to solve the puzzle of the different parities of the decay products of neutral Kaons. It was then observed in nuclear beta decay and later in charged pion decays.

Parity(P) $\bar{r} \rightarrow-\bar{r}$, Charge Conjugation(C) $q \rightarrow-q$ and Time Reversal(T) $\Delta t \rightarrow-\Delta t$ are Discrete Symmetries. In Figure 3, we illustrate $\mathrm{P}$ and $\mathrm{C}$ transformations, using as Reference the observed $\pi^{+} \rightarrow \mu^{+} v_{\mu}$ decay.

Whereas the P-transformed and C-transformed processes do not exist for pion decays, the CP-transformed decay $\pi^{-} \rightarrow \mu^{-} \bar{v}_{\mu}$ is observed with the same decay rate. We conclude that Parity, as well as Charge Conjugation, is maximally violated, whereas CP is a good symmetry for pion decays. 


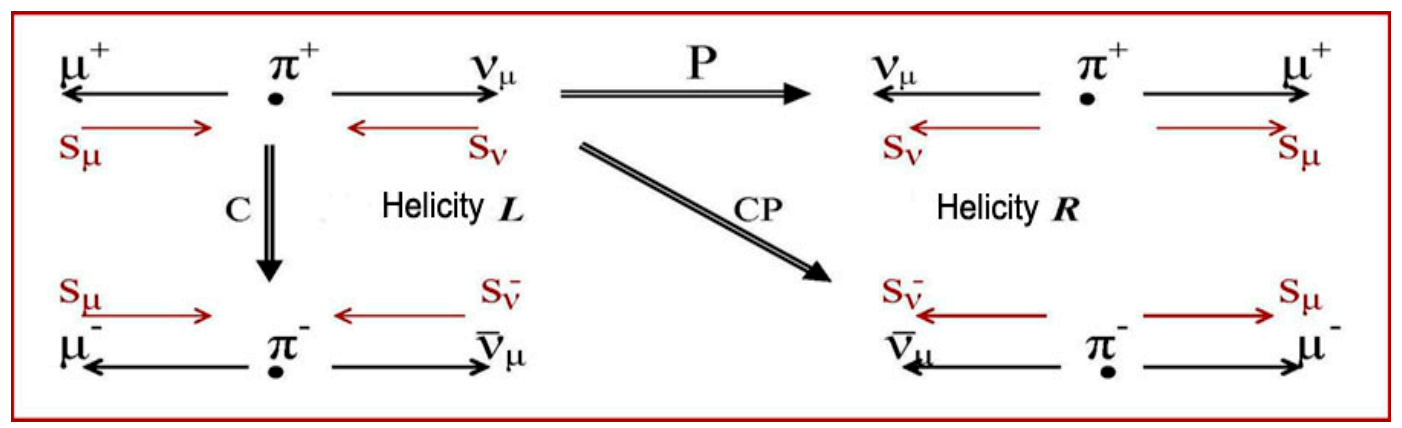

Figure 3. The $\mathrm{P}, \mathrm{C}$ and $\mathrm{CP}$ transformations in pion decays.

A chiral phenomenon is one which is not identical to its mirror image. The spin component of a particle along its momentum may be used to define a handedness, or helicity. For massless fermions, the helicity is the Lorentz invariant and this intrinsic property is the "Chirality". The invariance under Parity for a Dirac fermion state $\psi$ is called "Chiral Symmetry" and the transformation in Dirac space is implemented by the $\gamma_{5}$ Dirac matrix. Using Projectors, Left and Right chiral fermions, with definite chirality -1 and +1 , are provided by $\frac{1}{2}\left(1-\gamma_{5}\right) \psi, \frac{1}{2}\left(1+\gamma_{5}\right) \psi$. There are observables, like the vector and axial vector charges that conserve chirality of the fermions, whereas other observables, like the mass or dipole moments, connect the two chiralities.

In the Unified ElectroWeak Theory [8-10] based on the $\mathrm{SU}(2)_{\mathrm{L}} \times \mathrm{U}(1)_{\mathrm{Y}}$ gauge group, the fermion building blocks are not the Dirac fields $\psi$, but the Chiral Fields and the gauge group transformation distinguishes them: whereas the Left Field transforms as Doublets under SU(2) $\mathrm{L}$, the Right Field transforms as Singlets under $\mathrm{SU}(2)_{\mathrm{L}}$. We say that this Unified Field Theory is a Chiral Gauge Theory.

The ElectroWeak gauge group $\mathrm{SU}(2)_{\mathrm{L}} \times \mathrm{U}(1)_{Y}$ symmetry demands three gauge bosons $\mathrm{W}_{1}, \mathrm{~W}_{2}$, $\mathrm{W}_{3}$ of weak isospin from $\mathrm{SU}(2)_{L}$ and the $\mathrm{B}$ boson of weak hypercharge $\mathrm{Y}$ from $\mathrm{U}(1)_{\mathrm{Y}}$. The gauge symmetry is here broken by the Mass Terms and the Physical Fields with definite mass and charge are $\mathrm{W}^{ \pm}, \gamma, \mathrm{Z}$ given by

$$
\left(\begin{array}{l}
\gamma \\
Z
\end{array}\right)=\left(\begin{array}{cc}
\cos \theta_{w} & \sin \theta_{w} \\
-\sin \theta_{w} & \cos \theta_{w}
\end{array}\right)\left(\begin{array}{c}
B \\
W_{3}
\end{array}\right), \quad M_{z}=\frac{M_{W}}{\cos \theta_{w}}
$$

with $\theta_{W}$ as the weak mixing angle. The theory predicts the existence of Weak Neutral Currents mediated by the $\mathrm{Z}$ boson and they were discovered [11] by the Gargamelle Bubble Chamber Collaboration at CERN in 1973 with muon neutrino interactions without muons in the final state. Ten years later, in 1983, the UA1 and UA2 experiments in the $S \bar{p} p S$ Collider at CERN discovered the massive $\mathrm{W}, \mathrm{Z}$ Bosons as real particles reconstructed from their $\mathrm{W}^{+} \rightarrow 1^{+} v_{1}, \mathrm{Z} \rightarrow 1^{+} 1^{-}[12,13]$ decays. These CERN discoveries established the triumph of the Standard Model of ElectroWeak Interactions.

\subsection{GIM Mechanism: Need of Charm}

With u, d, s quarks only, the Cabibbo d-s Mixing [14] in the Charged Weak Current leads, by the $\mathrm{SU}(2)_{\mathrm{L}}$ symmetry of the Standard Model, to a Strangeness-Changing-Neutral Current at the tree level, implying, for example, fast $\mathrm{K}_{\mathrm{L}} \rightarrow \mu^{+} \mu^{-}$decay, against the experiment. In 1970, Glashow-Iliopoulos-Maiani [15] solved this problem with an additional fourth quark flavour, c, completing two families of quark doublets

$$
\left\{\begin{array}{l}
u \\
d
\end{array}\right\}\left\{\begin{array}{l}
c \\
s
\end{array}\right\}, \quad U=\left(\begin{array}{rr}
\cos \theta & -\sin \theta \\
\sin \theta & \cos \theta
\end{array}\right)
$$

and interpreting the Cabibbo Mixing as a Unitary Mixing Matrix $U$ in d-s space, exhibiting the mismatch between weak eigenstates and mass eigenstates, with Charged Currents relative to both $\mathrm{u}$ and $\mathrm{c}$ quarks. $\mathrm{SU}(2)_{\mathrm{L}}$ then dictates that Neutral Currents are governed by $\mathrm{U}^{+} \mathrm{U}=\mathrm{I}$, so they are Diagonal and Universal. Neutral Currents Flavour-Conserve at the tree level. At higher orders, 
Flavour-Changing-Neutral Currents can be induced from c-u mass difference. The $K_{L} \rightarrow \mu^{+} \mu^{-}$is suppressed—not only by higher orders-by the GIM additional factor $\left(\mathrm{m}_{\mathrm{c}}{ }^{2}-\mathrm{m}_{\mathrm{u}}{ }^{2}\right) / \mathrm{M}_{\mathrm{W}}{ }^{2}$.

The discovery [16,17] of the $c \bar{c} \mathrm{~J} / \psi$ meson in 1974 at BNL and SLAC is coined as the November Revolution of particle physics. Charmed $c \bar{d}, c \bar{s}, c u d \ldots$ hadrons were discovered later.

\subsection{The Third Family}

The existence of a third family of quarks was predicted in 1973 by Kobayashi and Maskawa [18] in order to incorporate CP Violation in the Standard Model, which is to be discussed in Section 4, using a generalization of the Cabibbo Mixing for weak charged currents. The $b \bar{b} \curlyvee$ meson was discovered in 1977 in FermiLab, and the B mesons later.

The Top Quark is the most massive of all observed elementary particles. With a mass of $172.44 \mathrm{GeV} / \mathrm{c}^{2}$, it weighs similar to an atom of tungsten. It decays by weak interaction $\mathrm{t} \rightarrow \mathrm{b}$ W with a lifetime of $5 \times 10^{-25} \mathrm{~s}$. Such a short life is $1 / 20$ of the timescale for Quark Hadronisation, allowing "bare" quark studies with its entire spin density matrix in the production, as well as in the decay.

The top quark was first indirectly "seen" with non-decoupling virtual quantum effects in $B^{0}-\bar{B}^{0}$ Mixing [19,20] measured by UA1 and ARGUS in 1987, in the universal Z boson self-energy [21] and in the specific $Z b \bar{b}$ vertex [22], the last two observed in the LEP experiments. The direct detection of top quarks was then made in 1995 at the $p \bar{p}$ Tevatron [23,24]. The $\mathrm{p} \mathrm{p}$ Collider LHC facility is at present a Top Quark Factory by means of its strong $g g \rightarrow t \bar{t}$ and weak $u \bar{d} \rightarrow t \bar{b}$ production mechanisms.

\subsection{Gauge Anomalies: Quark-Lepton Symmetry}

A Gauge Anomaly is a feature of quantum physics, a one-loop diagram, invalidating the gauge symmetry of a quantum field theory. All gauge anomalies must cancel out. Anomalies in gauge symmetries would destroy the required cancellation of unphysical degrees of freedom, such as a photon polarization in time direction.

Are gauge anomalies cancelled in the Standard Model? Anomalies appear in even D spacetime dimensions with Chiral fermions running in the loop with $n=1+D / 2$ vertices. For $D=4, n=3$, it corresponds to Vector-Vector-Axial couplings. The condition for cancellation involves the particle content and the relations among their couplings [25]: the symmetrized trace over the generators of the gauge group vanishes

$$
\operatorname{tr}\left(\left\{\tau_{i}, \tau_{j}\right\} \tau_{k}\right)=0
$$

Such a cancellation operates within each family of Quarks and Leptons, establishing an intriguing connection between the two sectors, announcing a Grand Unification. For the three families required to incorporate CP Violation, we then write the Symmetry between Quarks and Leptons as in Figure 4

$$
\left\{\begin{array}{l}
u \\
d
\end{array}\right\}\left\{\begin{array}{l}
c \\
s
\end{array}\right\}\left\{\begin{array}{l}
t \\
b
\end{array}\right\} \leftrightarrow\left\{\begin{array}{c}
v_{e} \\
e
\end{array}\right\}\left\{\begin{array}{c}
v_{\mu} \\
\mu
\end{array}\right\}\left\{\begin{array}{c}
v_{\tau} \\
\tau
\end{array}\right\}
$$

Figure 4. Quark-Lepton Symmetry requested for the cancellation of Equation (8).

\section{Discrete Symmetries CP, T, CPT}

As already discussed, the violation of Parity $\mathrm{P}$ and Charge Conjugation $\mathrm{C}$ had a profound impact in the way how the ElectroWeak Standard Model was formulated. In this section, we present the state-of-the-art with the observed Violations of $\mathrm{CP}$ and $\mathrm{T}$ and the tests on CPT.

\subsection{CP Violation}

CP Symmetry would imply that the Laws of Physics should be invariant in form when a particle is interchanged with its antiparticle $(C)$ while its spatial coordinates are inverted $(P)$. For the neutral 
Kaon system with $\Delta S=2$ Mixing $K^{0}-\bar{K}^{0}$ by weak interactions, the physical states of definite mass and lifetime $\mathrm{K}_{\mathrm{L}}$, $\mathrm{K}_{\mathrm{S}}$ should be $\mathrm{CP}$ eigenstates leading to Conservation Laws: the decay $\mathrm{K}_{\mathrm{L}} \rightarrow \pi \pi$ should be forbidden. Its unexpected observation [26] in 1964 opened the entire new field of CP Violation in Flavour Physics.

Can CP Violation be described in the Standard Model? In 1973, Kobayashi and Maskawa discovered [18] such a possibility by breaking the CP symmetry in the Standard Model Lagrangian by means of Cabibbo Mixing in the weak charged currents and enlarging the particle content of the theory. By going up to, at least, three families of fermions, the most general mismatch mixing matrix $U$ between weak and mass eigenstates for $d-s-b$ quarks contains a physical relative phase such that for antiquarks becomes its complex conjugate $\mathrm{U}^{*}$.

The precision measurements in the Kaon system with secondary Kaon beams [27,28] and at CPLEAR [29], and especially the observation of CPV in the $B_{d}$ system in the B-Factories [30,31] in 2001 prompted the conviction that the Cabibbo-Kobayashi-Maskawa (CKM) mechanism of Quark Mixing with three families of fermions is a good description of CPV in the SM (Standard Model). Recently, $\mathrm{CPV}$ in the charm system has been observed by the LHCb Collaboration [32] with a small magnitude as predicted by the SM. As seen, the origin of the Breaking is here advocated to be in the particle content of the theory.

All known laboratory experimental results on CP Violation for $\mathrm{K}, \mathrm{B}$ and D physics are in agreement with the Unitary Mixing Matrix paradigm U (CKM) with three active families of quarks. In Figure 5, the three families are displayed, and the corresponding "Unitarity Triangle" relation for $B_{d}$-physics represented

$$
\left\{\begin{array}{l}
u \\
d
\end{array}\right\}\left\{\begin{array}{l}
c \\
s
\end{array}\right\}\left\{\begin{array}{l}
t \\
b
\end{array}\right\}
$$

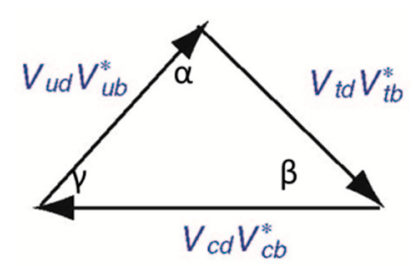

Figure 5. Three quark families and unitary triangle for $B_{d}$ physics.

One should notice: (i) the three upper $u, c, t$ quarks have to be involved; (ii) the three angles $\alpha, \beta$, $\gamma$ are $\mathrm{CP}$ violating observable phases, the first two involving the virtual $B^{0}-\bar{B}^{0}$ mixing through the heavier $\mathrm{t}$ quark whereas $\gamma$ is a signal of direct $\mathrm{CP}$ Violation in the decays of $\mathrm{c}$ and $\mathrm{u}$ quarks.

However, this Standard Model description of $\mathrm{CP}$ Violation is not enough to explain the Matter-Antimatter Asymmetry in the Universe.

\subsection{Time Reversal Violation}

A symmetry transformation $\mathrm{T}$ that changes the dynamics of a physical system into another with an inverted sense of time evolution is called Time Reversal (Reversal-in-Time). It is implemented in the space of states by an AntiUnitary Operator, implying that its study has to be made with asymmetries built under the exchange of in and out states.

The decay is an irreversible process indicating that a true TRV (Time Reversal Violation) observable, needing a definite preparation and filtering of the appropriate initial and final particle state, looks impossible for transitions in the case of decaying particles. A bypass to this NO-GO argument was found [33] using entangled systems of unstable particles with the ingredients: (i) the decay as a Filtering Measurement and (ii) entanglement implying the information transfer from the decayed particle to its living partner. For the entangled $B^{0}-\bar{B}^{0}$ system produced by $\mathrm{e}^{+} \mathrm{e}^{-}$collisions at the $\Upsilon(4 \mathrm{~S})$ peak, one may study the time dependence in meson transitions associated to the definite Flavour- $\mathrm{CP}$ 
eigenstate decay products. There are 2 (Flavour) $\times 2(\mathrm{CP}) \times 2$ (time ordering) $=8$ transitions of this kind which can be connected by different Separate Genuine T, CP, and CPT Symmetry Transformations.

In Figure 6, the experimental steps to measure the time dependent TRV asymmetry for the $\bar{B}^{0} \rightarrow B_{-}$ and $B_{-} \rightarrow \bar{B}^{0}$ meson transitions between Flavour and $C P$ eigenstates are provided, where $B_{-}$is the meson state filtered by the $\mathrm{J} / \psi \mathrm{K}_{\mathrm{S}}$ decay products.

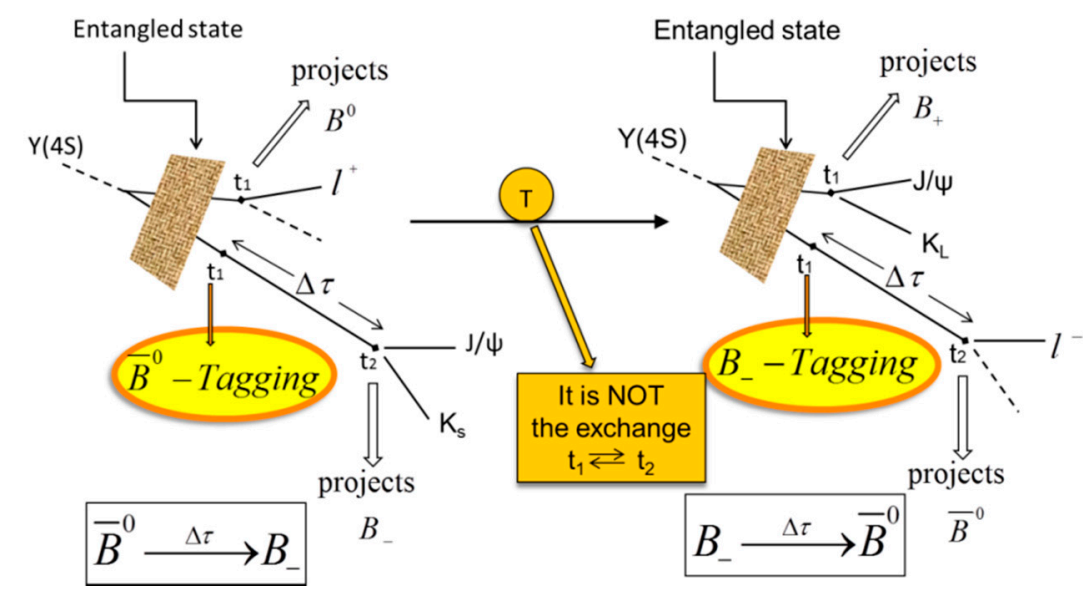

Figure 6. Experimental steps to observe TRV in the entangled $B_{d}$-system.

Using these concepts, the BABAR Collaboration observed [34] in 2012 is a true TRV effect with $14 \sigma$ significance. This discovery was a model-independent result, with the time-dependent form of the Intensity distribution as the only dynamical input, using the methodology previously defined in [35]. Its interpretation within the realm of the Weisskopf-Wigner approach for the effective Hamiltonian of the $B_{d}$-system, including CPT Violation, is full of interesting results [36] for the separation of genuine CPV and TRV observables.

For the neutral Kaon system, a search of TRV is ongoing at the KLOE-2 experiment in the DAPHNE-Factory, using entanglement as proposed in Ref. [37].

\section{3. $C P T$}

Although P, C, CP and T symmetries have been observed to be separately broken, there is no experimental evidence of CPT Violation. Its observation would imply a change of paradigm to beyond local QFT. There is, however, nothing against a possible breaking of CPT Invariance at the level of Quantum Mechanics. Most sensitive limits come from the neutral Kaon $K^{0}-\bar{K}^{0}$ mass difference and the frequencies of the antihydrogen transitions.

I discuss tests of the CPT symmetry using the semileptonic decays of neutral Kaons. Assuming the $\Delta S=\Delta \mathrm{Q}$ rule, as implied by the description of flavoured mesons in terms of quarks, the allowed transitions are pictured in the diagrams.

In the flavour states $K^{0}, \bar{K}^{0}$, the effective Hamiltonian in a Weisskopf-Wigner approach includes a non-diagonal CPV parameter and a diagonal CPTV parameter, so that the states with definite time evolution are

$$
\begin{aligned}
& \left|K_{s}\right\rangle=\frac{1}{\sqrt{2\left(1+\left|\epsilon_{s}\right|^{2}\right)}}\left(\left(1+\epsilon_{s}\right)\left|K^{0}\right\rangle+\left(1-\epsilon_{s}\right)\left|\bar{K}^{0}\right\rangle\right) \\
& \left|K_{L}\right\rangle=\frac{1}{\sqrt{2\left(1+\left|\epsilon_{L}\right|^{2}\right)}}\left(\left(1+\epsilon_{L}\right)\left|K^{0}\right\rangle-\left(1-\epsilon_{L}\right)\left|\bar{K}^{0}\right\rangle\right)
\end{aligned}
$$

where

$$
\epsilon_{\frac{S}{L}}=\epsilon \pm \delta
$$

where " $E$ " is the parameter describing CPV whereas " $\delta$ " is the parameter describing CPTV. 
At CPLEAR, the preparation of an initial quantum state of flavour was used. With this tag, the following time-dependent combination of semileptonic decay rates was constructed as an asymmetry

$$
\left\{\begin{aligned}
A_{\delta}(\tau)= & \frac{\bar{R}+(\tau)-\alpha R_{-}(\tau)}{\overline{\bar{R}}(\tau)+\alpha R_{-}(\tau)}+\frac{\bar{R}-(\tau)-\alpha R_{+}(\tau)}{\bar{R}-(\tau)+\alpha R_{+}(\tau)} \\
R_{+(-)}(\tau)= & R\left(K_{t=0}^{0} \rightarrow\left(e^{+(-)} \pi^{-(+)} v\right)_{t=\tau}\right) \\
\bar{R}_{-(+)}(\tau)= & R\left(\bar{K}_{t=0}^{0} \rightarrow\left(e^{-(+)} \pi^{+(-)} v\right)_{t=\tau}\right) \\
& \alpha=1+4 \operatorname{Re}\left(\epsilon_{L}\right)
\end{aligned}\right.
$$

At times outside the interference region, i.e., longer than the short lifetime of $K_{S}$, this asymmetry becomes a pure CPT test and determined by the Re $(\delta)$ parameter as

$$
A_{\delta}\left(\tau \gg \tau_{S}\right)=8 \operatorname{Re}(\delta)
$$

It is worth noting that $\operatorname{Re}(\delta)$ does not need any absorptive part and it is determined by the difference of diagonal mass terms of $K^{0}$ and $\bar{K}^{0}$. The result of the measurement of the asymmetry (11) by the CPLEAR Collaboration [38] is

$$
\operatorname{Re}(\delta)=(0.30 \pm 0.33 \pm 0.06) \times 10^{-3}
$$

where the first error is statistical and the second systematic.

Its interpretation in terms of the mass matrix elements of the $K^{0}-\bar{K}^{0}$ system gives the upper bound

$$
\left|m_{K^{0}}-m_{\bar{K}^{0}}\right| / m_{K}<10^{-18}
$$

If we take, as a figure of merit, this fractional difference between the masses of a particle and its antiparticle, the result (14) appears to be the best CPT Violation Limit.

At the $\Phi$-Factory DAPHNE, the entanglement between the two neutral Kaon states induced by their antisymmetric exchange allows for a separate selection of observable asymmetries from either $\mathrm{K}_{\mathrm{L}}$ or $\mathrm{K}_{\mathrm{S}}$ states. The Charge Asymmetry from these states is obtained by

$$
A_{S, L}=\frac{\Gamma\left(K_{S, L} \rightarrow \pi^{-} e^{+} v\right)-\Gamma\left(K_{S, L} \rightarrow \pi^{+} e^{-\bar{v}}\right)}{\Gamma\left(K_{S, L} \rightarrow \pi^{-} e^{+} v\right)+\Gamma\left(K_{S, L} \rightarrow \pi^{+} e^{-} \bar{v}\right)}=2[\operatorname{Re}(\epsilon) \pm \operatorname{Re}(\delta)-\operatorname{Re}(\mathrm{y})]
$$

where, in addition to the CPV and CPTV parameters in the Hamiltonian matrix, a possible CPTV in the flavour decay amplitudes of Figure 7, parameterised by " $y$ ", is included. As seen, the difference between these two Charge Asymmetries $A_{S}-A_{L}$ is the ability to isolate CPTV effects in the diagonal matrix elements of the Mass Matrix.
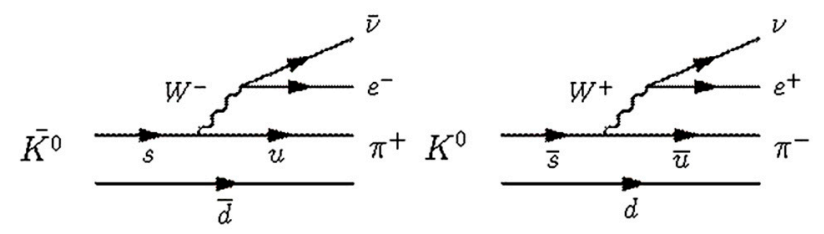

Figure 7. Two decays are allowed according to elementary Quarks ( $\Delta S=\Delta Q$ rule).

The value of $A_{L}$ is well known from hadronic machines, with the best precise result from the $\mathrm{KTeV}$ Collaboration [39]

$$
\mathrm{A}_{\mathrm{L}}=\left(3.332 \pm 0.058_{\text {stat }} \pm 0.047_{\text {syst }}\right) \times 10^{-3}
$$


The measurement of $A_{S}$ was undertaken in the first period of the KLOE experiment with the result [40]

$$
\mathrm{A}_{\mathrm{S}}=\left(1.5 \pm 9.6_{\text {stat }} \pm 2.9_{\text {syst }}\right) \times 10^{-3}
$$

An important improvement of this result is expected at KLOE-2.

A direct test of CPT in neutral Kaon transitions has been proposed. EPR correlations at a $\Phi$-Factory can be exploited to study CPT-conjugated transition involving Flavour $K^{0}, \bar{K}^{0}$ states and the orthogonal $K_{+}, K_{-}$states filtered by CP eigenstate decay products $\pi \pi$ and $\pi^{0} \pi^{0} \pi^{0}$.

For a CPT-symmetry test, one has a priori four possible comparisons of transitions as shown in Table 1

Table 1. Reference transitions and their CPT-transformed transitions.

\begin{tabular}{cccc}
\hline \multicolumn{2}{l}{ CPT Symmetry Test } & & \\
\hline \multicolumn{1}{c}{ Reference } & \multicolumn{3}{c}{ CPT-conjugate } \\
\hline Transition & Decay products & Transition & Decay products \\
\hline$K^{0} \rightarrow K_{+}$ & $\left(l^{-}, \pi \pi\right)$ & $K_{+} \rightarrow \bar{K}^{0}$ & $\left(3 \pi^{0}, l^{-}\right)$ \\
$K^{0} \rightarrow K_{-}$ & $\left(l^{-}, 3 \pi^{0}\right)$ & $K_{-} \rightarrow \bar{K}^{0}$ & $\left(\pi \pi, l^{-}\right)$ \\
$\bar{K}^{0} \rightarrow K_{+}$ & $\left(l^{+}, \pi \pi\right)$ & $K_{+} \rightarrow K^{0}$ & $\left(3 \pi^{0}, l^{+}\right)$ \\
$\bar{K}^{0} \rightarrow K_{-}$ & $\left(l^{+}, 3 \pi^{0}\right)$ & $K_{-} \rightarrow K^{0}$ & $\left(\pi \pi, l^{+}\right)$ \\
\hline
\end{tabular}

For the most interesting $\Delta \mathrm{t}$ region in the transition probabilities, with $\Delta \mathrm{t}$ larger than the $\mathrm{K}_{\mathrm{S}}$ lifetime, one obtains [41]

$$
\begin{aligned}
& R_{2}\left(\Delta t \gg \tau_{S}\right)=P\left[K^{0} \rightarrow K_{-}\right] \frac{}{P\left[K_{-} \rightarrow \bar{K}^{0}\right]}=1-4 \operatorname{Re}(\delta) \\
& R_{4}\left(\Delta t \gg \tau_{S}\right)=P\left[\bar{K}^{0} \rightarrow K_{-}\right] \frac{}{P\left[K_{-} \rightarrow K^{0}\right]}=1+4 \operatorname{Re}(\delta)
\end{aligned}
$$

One cannot imagine better clearest tests of CPT-symmetry in transitions as those of Equation (18). These tests are feasible at KLOE-2.

Up to now, we have considered CPTV effects associated with the incompatibility of the CPT-operator with the Hamiltonian $\mathrm{H}$, i.e., the non-invariance of $\mathrm{H}$ under CPT [CPT, $\mathrm{H}] \neq 0$. There is a possible second source of CPT-breaking induced by the quantum gravity vacuum: the $\omega$-effect.

In the presence of decoherence and CPT breaking induced by quantum gravity effects, the CPT operator is "ill-defined", and the definition of the particle-antiparticle states could be modified. This in turn could induce a weakening of the Entanglement [42] imposed by Bose statistics as an EPR correlation

$$
\begin{array}{r}
|i\rangle\left(\left|K^{0}\right\rangle\left|\bar{K}^{0}\right\rangle-\left|\bar{K}^{0}\right\rangle\left|K^{0}\right\rangle\right)+\omega\left(\left|K^{0}\right\rangle\left|\bar{K}^{0}\right\rangle+\left|\bar{K}^{0}\right\rangle\left|K^{0}\right\rangle\right) \\
\propto\left(\left|K_{s}\right\rangle\left|K_{L}\right\rangle-\left|K_{L}\right\rangle\left|K_{S}\right\rangle\right)+\omega\left(\left|K_{s}\right\rangle\left|K_{s}\right\rangle-\left|K_{L}\right\rangle\left|K_{L}\right\rangle\right)
\end{array}
$$

In some microscopic models of space-time foam [43] with stochastic fluctuations of defect recoils in the propagation of particles, the magnitude of the $\omega$-parameter in Equation (19) can reach values $\omega \sim 10^{-4}-10^{-5}$.

The maximum sensitivity to $\omega$ is expected for a symmetric decay pair of the entangled state $\mathrm{f} 1=\mathrm{f} 2=\pi^{+} \pi^{-}$, which is CP-violating from the dominant antisymmetric state of Equation (19). As a consequence, one gets a fantastic enhancement of the $\omega$-effect from the "wrong" component in Equation (19) as $\omega / \varepsilon$, where $\varepsilon$ is the small CPV parameter in K-physics. The higher luminosity of present DAPHNE and a good time resolution at KLOE-2 allow reaching an interesting sensitivity to this most fascinating $\omega$-effect.

In the last few years, a new actor appeared: aggregate antimatter for tests of CPT. Antihydrogen experiments pursue very precise measurements of atomic transition frequencies. The CERN accelerator complex produces antiprotons through $p+p \rightarrow p+p+p+\bar{p}$ and, since 2000, the Antiproton 
Decelerator (AD) operates like an all-in-one machine. The ELENA Project is expected to be in operation until 2021, with the Call of Proposals already completed in 2019. Among the AD/ELENA experiments, the ALPHA Collaboration is involved in the 1S-2S spectroscopy in antihydrogen, and the ASACUSA Collaboration includes in its programme the antihydrogen hyperfine splitting (HFS).

In hydrogen, the $1 S-2 S$ transition corresponds to a two-photon channel with $\lambda=243 \mathrm{~nm}$, measured with a precision $\Delta \mathrm{f} / \mathrm{f}=10^{-14}$. The ground state hyperfine splitting corresponds to the famous $\lambda=21 \mathrm{~cm}$ line, with $\mathrm{f}=1.4 \mathrm{GHz}$ and measured with a precision $\Delta \mathrm{f} / \mathrm{f}=10^{-12}$. The theoretical uncertainty, due to the proton structure, is at the level of $10^{-6}$, however.

The first spectroscopy results with antihydrogen at AD were obtained by ALPHA for 1S-2S $\left(\sim 10^{-12}\right)[44]$ and for HFS $\left(\sim 10^{-4}\right)$ [45]. New techniques in spectroscopy and antiproton beams promise higher accuracy for HFS by ASACUSA [46], and much more is expected to come with ELENA.

\section{Neutrinos}

The last two decades have seen a revolution in neutrino physics with the discovery of, and precision studies on, flavour oscillations in atmospheric [47], solar [48], reactor [49] and accelerator [50] neutrinos. These phenomena are interpreted in terms of non-vanishing masses and flavour mixing, the unitary PMNS matrix [51,52] describing the mismatch between flavour and mass eigenstates. Global fits to all observable quantities provide better and better determination of the two mass differences $\Delta m_{21}^{2}$ and $\left|\Delta m_{31}^{2}\right|$, as well as the three mixing angles [53-55].

The most fundamental pending questions are the Dirac-Majorana confusion on the nature of neutrinos and their absolute mass scale, properties studied by means of non-accelerator methods, as well as the novel challenges for the next-generation neutrino flavour oscillation experiments like T2HK [56] and DUNE [57] using terrestrial accelerators. Above all, once it is known that the three mixing angles are non-vanishing [58-61], they should answer whether the lepton sector of elementary particles also incorporates $\mathrm{CP}$ violation, opening the door to concepts able to explain the matter-antimatter asymmetry of the Universe through leptogenesis [62] at higher energy scales. An additional open problem is the ordering of the neutrino mass spectrum, with the so-called Normal and Inverted Hierarchies.

\subsection{The Energy Crisis}

Before 1930, the only subatomic particles known were the electron [63] and the proton [64]. Together with a still-developing quantum theory, these two elementary particles were enough to describe the atomic spectrum of hydrogen. The structure of atomic nuclei [65] was initially thought of in terms of bounded protons and electrons, especially motivated by the identification of $\beta$ radioactivity [66] with electron emission [67],

$$
{ }_{Z}^{A} X \rightarrow{ }_{Z+1}^{A} Y+e^{-}
$$

as is the case of ${ }_{6}^{14} C$, decaying into ${ }_{7}^{14} N$ with a half-life of about 5730 years. The conservation of energy and momentum in this two-body decay predicts a definite value for the emitted electron energy,

$$
E_{e}=\frac{m_{X}^{2}-m_{Y}^{2}-m_{e}^{2}}{2 m_{X}}
$$

Lise Meitner and Otto Hahn in 1911 [68] and Jean Danysz in 1912 [69] obtained the first hint that $\beta$ particles have a continuous spectrum, against the energy conservation rule leading to Equation (21). Further evidence of the violation of energy conservation was given by James Chadwick in 1914 [70], measuring that the spectrum was continuous. In addition, molecular band spectra established [71] that the nuclear spin of Nitrogen-14 is 1, also implying the violation of angular momentum conservation in the process (20), which would thus involve the spins $0 \rightarrow 1+\frac{1}{2}$. Dedicated studies in the period 1920-1927 by Charles Drummond Ellis and others [72-74] finally demonstrated beyond any doubt that the spectrum is continuous, as shown in Figure 8. 


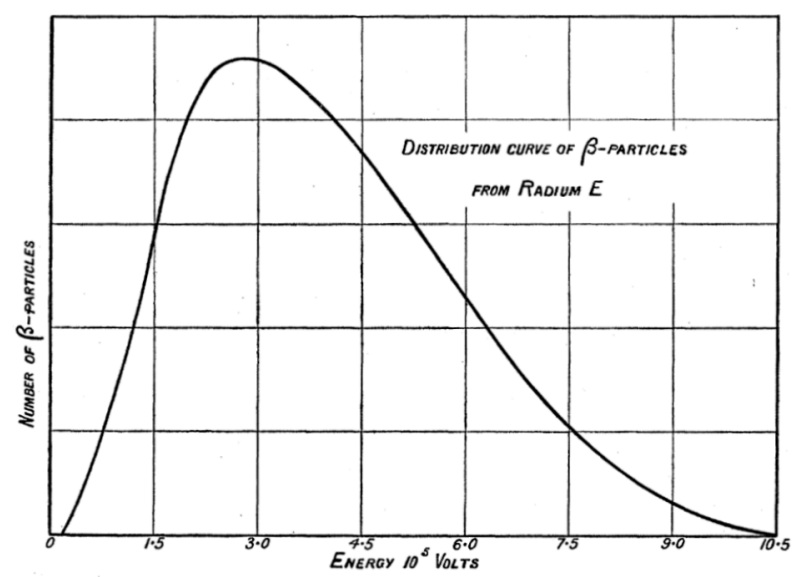

Figure 8. $\beta$-decay electron energy spectrum [64].

In a desperate reaction to these experimental facts, Niels Bohr postulated that the conservation of energy was true in a statistical sense only [75]. These $\beta$ decay puzzles of non-conservation of energy and angular momentum were on the forefront of the open problems in the fundamental physics endeavour.

Indeed, the fact that the measured energy ranged from zero to a maximum given by its nominal value in Equation (21) cannot be explained in this framework. Furthermore, the nuclear models with A protons and A-Z electrons were not able to explain the spin of the nuclei, which for, e.g., ${ }_{7}^{14} \mathrm{~N}$ should have been half-odd instead of the measured integer value. In this context, both conservation of energy and angular momentum seemed to be failing.

In 1930, Wolfgang Pauli wrote the famous letter of the 4th of December, addressed to the "Dear Radioactive Ladies and Gentlemen" participating in the Tübingen Conference: he proposed the existence of a hitherto unobserved spin-1/2 neutral particle with a small mass, no greater than $1 \%$ the mass of a proton, which he called a "neutron". In his words, this was a desperate remedy to save the exchange theorem of statistics and the law of conservation of energy. Assuming that this particle was emitted together with the electron in $\beta$ processes,

$$
{ }_{6}^{14} \mathrm{C} \rightarrow{ }_{7}^{14} \mathrm{~N}+e^{-}+v
$$

the law of conservation of energy could successfully explain the spectrum in Figure 8, and angular momentum conservation could describe the $0 \rightarrow 1+\frac{1}{2}+\frac{1}{2}$ transition too. Moreover, the presence of this hypothetical neutral particle in the nucleus, with the same number as electrons, could also solve the problem of nuclear spin.

Pauli excused his participation in the conference: «I cannot appear in Tübingen personally since I am indispensable here in Zurich because of a ball on the night of the 6th to 7th of December». The fact that this hypothetical particle had not been observed in $\beta$ decay forced it to interact very weakly, to the point that Pauli himself believed that it would never be observed. Unlike nowadays, when theoreticians have no problem in proposing particles whose observation is unfeasible, Pauli avoided claiming his solution in a scientific journal.

The nuclear spin problem was soon solved: James Chadwick discovered the neutron in 1932 [76]. Its spin $1 / 2$ and mass similar to proton mass opened the door to the understanding of the atomic nucleus in terms of protons and neutrons. Its heavy mass, however, made it clear that this particle could not be identified as Pauli's "neutron", and indeed the problems with conservation of energy and angular momentum in $\beta$ decays were still present.

The description of the subatomic world in terms of protons, electrons and neutrons lived a short life. A few months after Chadwick's discovery of the neutron, Carl D. Anderson took the photograph of a positron [77], finding the first evidence on the existence of antimatter. In 1934, Enrico Fermi 
incorporated Pauli's neutral particle, which he named "neutrino", together with all of these particles into his theory of $\beta^{ \pm}$decays [78], described as the subatomic processes

$$
\begin{aligned}
& n \rightarrow p+e^{-}+\bar{v} \\
& p \rightarrow n+e^{+}+v
\end{aligned}
$$

His theory successfully predicted the spectrum shapes, and provided a framework to calculate their lifetimes, supporting Pauli's proposed particle.

Using Fermi's theory, Hans Bethe and Rudolf Peierls estimated in 1934 [79] the mean absorption length of neutrinos in solid matter, a value larger than $10^{14} \mathrm{~km}$ : the expected cross section of neutrinos with matter was so low that they could traverse the Earth without any interaction. They concluded that «it is absolutely impossible to observe processes of this kind». Due to this low interaction rate, it was not until 1956 that the Cowan-Reines experiment [80] at the Savannah River Nuclear Reactor proved the existence of the neutrino. Antineutrinos created in the nuclear reactor by the $\beta$ decay were subsequently detected by the process

$$
\bar{v}+p \rightarrow n+e^{+}
$$

in a large water tank. The signature of the process is the two $511 \mathrm{keV}$ photons from the positron annihilation, in coincidence with the nuclear photon

$$
n+{ }^{108} \mathrm{Cd} \rightarrow{ }^{109} \mathrm{Cd}+\gamma
$$

due to the de-excitation of the ${ }^{109} \mathrm{Cd}$ nucleus after the neutron was captured by cadmium chloride molecules $\left(\mathrm{CdCl}_{2}\right)$ dissolved in the water.

Beyond the theoretical motivation for such a neutral particle proposed by Pauli and bolstered by Fermi, unquestionable evidence for its existence had been provided by Reines and Cowan. As such, the neutrino was born.

\subsection{Lepton Flavours}

With the discovery of the muon [81,82], an initial problem appeared with its insertion in the Fermi theory of charged current weak interactions. Indeed, the muon was so unexpected that, regarding its discovery, Isidor Isaac Rabi famously quipped «Who ordered that? ». A decade before the V-A theory [83], Bruno Pontecorvo discussed [84] the "universality" of Weak Interactions for nuclear $\beta$-decay processes together with those with muons and neutrinos. He introduced muon capture by nuclei and compared it with the probability for electron capture.

The idea of different neutrinos $v_{\mu} \neq v_{e}$ appeared published in 1959 by Pontecorvo [85] and, ever more important, in the proposal he made for the generation of a neutrino beam from pion decay [86].

In 1962, there was already a hint towards the different nature of the electron and muon neutrinos, originating from the $\mu \rightarrow e \gamma$ decay. An estimation of its branching ratio in the V-A theory with the $\mathrm{W}$ boson had yielded, if $v_{\mu}=v_{e}$, the value [87] $\mathrm{R}_{\text {th }} \sim 10^{-4}$, whereas a limit $\mathrm{R}_{\exp }<10^{-8}$ was found [88]. The Brookhaven experiment [89] was the first one involving high-energy neutrinos from pion decay. Using about $10^{14}$ muon antineutrinos from $\pi^{-}$, the experiment detected 29 events of the expected

$$
\bar{v}_{\mu}+p \rightarrow \mu^{+}+n
$$

and no events of the forbidden

$$
\bar{v}_{\mu}+p \rightarrow e^{+}+n
$$

The demonstration that $v_{\mu} \neq v_{e}$ was a great event in physics and thus two lepton families were demonstrated:

$$
\text { 1. }\left(v_{e}, e\right) \quad \text { 2. }\left(v_{\mu}, \mu\right)
$$


After the discovery of the third charged lepton $\tau$ [90], the existence of (at least) a third neutrino was to be expected. The third neutrino $v_{\tau}$ was directly observed in 2000 in an experiment at Fermilab performed by the DONUT collaboration [91]. Using the Tevatron beam, a $v_{\tau}$ beam was mainly produced from $\tau$ decay, with the $\tau$ produced in the leptonic decay of a $D_{S}$ meson. These $v_{\tau}$ were detected by means of the reaction

$$
v_{\tau}+n \rightarrow p+\tau^{-}
$$

by identifying the $\tau$ lepton as the only lepton created in the interaction vertex.

Two complementary methods for the determination of the number of neutrino species led to the highlighted LEP $\mathrm{e}^{+} \mathrm{e}^{-}$Collider legacy at CERN [92]. The invisible width of the $\mathrm{Z}$ boson

$\Gamma_{i n v}=N_{v} \Gamma_{v}$ is not measurable directly, but the visible cross section depends on the number of neutrino species $\mathrm{N}_{v}$ as

$$
\sigma=\frac{4 \pi s}{M_{Z}^{2}} \Gamma_{e e} \frac{\Gamma_{Z}-N_{v} \Gamma_{v}}{\left(s-M_{Z}^{2}\right)^{2}+\Gamma_{Z}^{2} M_{Z}^{2}}
$$

The hadronic cross-section is shown in Figure 9, from which LEP could determine the existence of three (and only three) light active neutrinos.

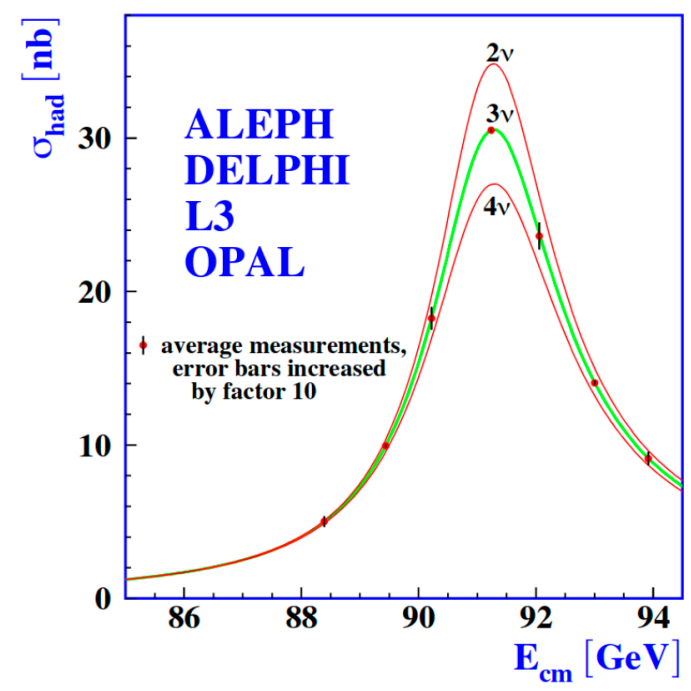

Figure 9. Measurements of the hadron production cross-section around the $\mathrm{Z}$ resonance. The curves indicate the predicted cross-section for two, three and four neutrino species with. SM couplings and negligible mass [92].

An alternative at LEP was the measurement of the cross-section for $e^{+} e^{-} \rightarrow \gamma Z \rightarrow \gamma v \bar{v}$ detecting the photon plus nothing else. This cross section, normalized to $\gamma \mu \mu$, is a known function of $N_{v}$, and consistently reproduced the previous answers: there are three light active neutrinos.

\subsection{Neutrino Mass and Mixing: Oscillations}

Parity violation in processes involving neutrinos, like $\beta$ decay, is automatic if neutrinos have a definite helicity. This fact led to the advent of the two-component theory-introduced by Hermann Weyl in 1929 [93] — if neutrinos are exactly massless, for which the Dirac equation

$$
\mathrm{i} \gamma^{\mu} \partial_{\mu} v_{L}(\mathrm{x})-m_{v} v_{R}(\mathrm{x})=0
$$

decouples. Non-conservation of parity was first observed in 1957 with Wu's experiment [94], measuring an asymmetry in the electron angular distribution emitted from the $\beta$ decay of polarized ${ }_{27}^{60} \mathrm{Co}$. In 1958 , the celebrated Goldhaber experiment [95] proved that the neutrino helicity is -1 , using conservation of angular momentum in a selected electron capture transition in ${ }_{63}^{152} \mathrm{Eu}$ leading to an excited nuclear state 
of ${ }_{62}^{152} \mathrm{Sm}$ - the measurement of the circular polarization of the de-exciting $\gamma$ ray fixed the neutrino helicity.

Even though this picture was consistent for massless neutrinos with definite helicity, the universal V-A theory of charged-current weak interactions was also formulated in 1958 [83], extending Fermi's theory of $\beta$ decay into a picture where left-handed chiral fields enter for all fermions, whether they are neutrinos or not. Thus, there is no rationale why neutrinos should be special and massless. Still, contrary to other fermions, neutrinos have no electric charge, so they present unique possibilities in the explanation of the origin of their masses. Whether these possibilities can be realized depends on the existence of a lepton charge that distinguishes $v$ and $\bar{v}$. This is the most important open question even today.

Already by 1946, Pontecorvo asked the question whether antineutrinos produced from $\beta$ decay in reactors could produce electrons [96]. The negative result in 1959 of the Davis experiment [97] for

$$
\bar{v}+{ }_{17}^{37} \mathrm{Cl} \rightarrow e^{-}+{ }_{18}^{37} \mathrm{Ar}
$$

suggested the existence of definite lepton numbers with values $L_{e^{-}}=L_{v}=1$ and $L_{e^{+}}=L_{\bar{v}}=-1$. Such a flavour charge would imply that neutrinos are not neutral particles, and so their mass could only be described with the same theories as all other fermions. The genius of Pontecorvo was the first to realize that this conclusion was referring to interacting neutrinos, and that there was still a possibility for neutrinos to acquire a Majorana mass.

The theory of Majorana particles had been introduced in 1937 by Ettore Majorana [98], who noticed that there are solutions of the Dirac equation for neutral particles with only two degrees of freedom: «the meaning of the Dirac equations is somewhat modified and there is no longer any reason to speak of negative-energy states nor to assume, for any other types of particles, especially neutral ones, the existence of antiparticles, corresponding to the "holes" of negative energy".

Forbidden for the other elementary fermions due to exact electric charge conservation, a Majorana mass for neutrinos is a priori allowed if the mass terms violate the global lepton number by two units. In this case, the states of neutrinos with definite Majorana mass would be a linear superposition of weak interacting neutrinos with opposite lepton charges. The global lepton number would then be undefined for neutrinos with definite Majorana mass. Even more; one can have massive neutrinos with the active (left-handed) chiral component $v_{\mathrm{L}}$ only and the sterile (right-handed) component $v_{\mathrm{R}}$ is not needed. Contrary to Dirac fermions, Majorana fermions have only two degrees of freedom, the neutrino of left-handed chirality and its conjugate, $v_{L}^{c}$. The states of definite mass and helicity, which are compatible observables, are the left-handed with a relative $\mathrm{m} / \mathrm{E}$ component of the conjugate, and its orthogonal.

It is astonishing that the early ideas on neutrino mixing came to these reasonings. In 1957, Pontecorvo writes [51]: «If the theory of two-component neutrinos were not valid, and if the conservation law for "neutrino charge" took not place, neutrino-antineutrino transitions would be possible». In this statement, one finds the two essential ingredients for oscillations: neutrino mass and mixing. He calculated [99] the survival probability of active neutrinos in a model of two Majorana neutrinos, one active, the other sterile, and suggested that the Cowan-Reines experiment should be repeated as function of the baseline for the detector. This kind of measurement in reactors was only performed for the first time in 2005 in the KAMLAND experiment [100].

In 1962, in the context of the Nagoya model of Baryons as bound states of neutrinos and "a new sort of matter" vector boson, Ziro Maki, Masami Nakagawa and Shoichi Sakata [52] introduced a mixing between two neutrinos to form the "true neutrinos" in these baryons. The objective was the explanation of the smallness of the leptonic decay rate of the hyperons, later explained by the Cabibbo mixing [14]. Although these ideas were not connected to neutrino oscillation phenomena, the concept of quantum mixings between neutrino states was there.

In 1967, after the discovery of the muon neutrino, Pontecorvo discussed [101] the phenomenology of neutrino oscillations in modern views, including the flavour transitions $v_{e} \rightarrow v_{\mu}$ and the Majorana 
transitions $v_{e} \rightarrow\left(v_{e}^{c}\right)_{L}$ and $v_{\mu} \rightarrow\left(v_{\mu}^{c}\right)_{L}$. Among other subjects, he applied this study to solar neutrino oscillations. At that time, Raymond Davis started his famous experiment on the detection of solar neutrinos [102]. They were detected via the observation of the reaction

$$
v+{ }_{17}^{37} \mathrm{Cl} \rightarrow e^{-}+{ }_{18}^{37} \mathrm{Ar}
$$

The results, measuring a $v_{\mathrm{e}}$ flux significantly smaller than expected from solar models, created the solar neutrino problem.

The other grand historical problem in the neutrino field, the atmospheric neutrino problem, appeared in 1988 with the measurement of an anomalously small $v_{\mu}$ flux at Kamiokande [103]. The understood origin of atmospheric neutrinos from cosmic rays

$$
\pi \rightarrow \mu v_{\mu}, \quad \mu \rightarrow e v_{e} v_{\mu}
$$

predicted a precise ratio of muon to electron neutrinos of 2:1, whereas the smaller muon neutrino flux observed lead to a ratio of 1.2.

These two problems were eventually solved with the consolidation of the oscillation paradigm. The discovery of neutrino oscillations in 1998 by Super Kamiokande [47] solved the atmospheric neutrino problem together with the later corroboration with solar neutrinos by SNO [48], solving the solar neutrino problem, marking the birth of the modern era in neutrino physics.

Historically, it is spectacular that the fundamental concepts of e- $\mu$ universality, flavour families, neutrino mixing and oscillations were understood in a period in which the prevailing idea was that of massless neutrinos. In the centenary of the birth of Pontecorvo, it was concluded [104] that it is fair to name the neutrino mismatch of flavour states with mass eigenstates as the PMNS Mixing Matrix.

\subsection{Genuine CP Violation}

Neutrino flavour oscillations have determined neutrino mass differences as oscillation frequencies and the mismatch mixings between flavour and mass eigenstates. Upon learning that the three mixing angles are non-vanishing, novel challenges include the search for $\mathrm{CP}$ Violation in the lepton sector. A recent constraint by T2K [105] on the allowed CP phase in the PMNS Mixing Matrix, at the level of $3 \sigma$, has been highly celebrated. This result is a prelude of the expectations for the next-generation experiments like T2HK [56] and DUNE [57]. The incorporation of CP Violation in the lepton sector would open the door to concepts able to explain the matter-antimatter asymmetry of the universe through leptogenesis [62] at higher energy scales. A second open problem is the ordering of the neutrino mass spectrum, with the so-called Normal or Inverted Hierarchy.

Long baseline neutrino oscillation experiments propagate neutrinos from the source, created as muonic flavour, to the detector through the Earth's mantle. The observation of CP violation needs an appearance experiment to a different flavour, and the "suppressed" transition to the electronic flavour is favoured. The corresponding CP violation asymmetry, defined in terms of the transition probabilities for neutrinos and antineutrinos, is an odd function of $\mathrm{L} / \mathrm{E}$, with $\mathrm{L}$ the baseline and $\mathrm{E}$ the relativistic neutrino energy, if the propagation takes place in vacuum. Independent of particular theoretical frameworks, this observable is a bona-fide direct proof of $\mathrm{CP}$ violation. However, in actual experiments, the propagation takes place in matter, which is $\mathrm{CP}$-asymmetric, and a fake $\mathrm{CP}$ violation is caused through the different interaction of electron neutrinos and antineutrinos with the electron density of ordinary matter $[106,107]$. This complication in the quest for direct evidence of fundamental CP violation is widely recognized, and some observables [108-110] have been tried for its separation. On the other hand, matter-induced terms are welcome in order to obtain information on the neutrino mass hierarchy. The restoration of the idea that direct evidence of symmetry violation means the measurement in a single experiment of an observable odd under the symmetry has been solved recently [111,112]. The concept exploited is based on the fact that genuine and matter-induced CP violation have opposite behaviors [113] under the other discrete symmetries of Time-Reversal $\mathrm{T}$ and 
$\mathrm{CPT}$; whereas genuine $\mathrm{CP}$ violation is odd under $\mathrm{T}$ and even under $\mathrm{CPT}$, the matter effect is $\mathrm{T}$-even and CPT-odd. Although they are well separated in the effective Hamiltonian, in general, they are not in the experimental observables and, in particular, in the CP asymmetry. The ideal way to solve this problem would be the independent measurement of T-odd [114] and CPT-odd asymmetries, but this route requires sources of electron neutrinos and antineutrinos above the muon mass energies, which is, at present, unavailable for accelerator facilities. As an alternative, the solution consists in disentangling these two components, genuine and matter-induced $\mathrm{CP}$ violation, in the observable $\mathrm{CP}$ asymmetry.

A Disentanglement Theorem [111] has been proved for any flavour transition. Neutrino oscillation $\mathrm{CP}$-violating asymmetries in matter have two disentangled components: (i) a CPT-odd T-invariant term, non-vanishing if there is interaction with matter; (ii) a T-odd CPT-invariant term, non-vanishing if there is genuine $\mathrm{CP}$ violation. As a function of the baseline, these two terms are distinct L-even and L-odd observables to separately test (i) matter effects sensitive to the neutrino hierarchy and (ii) genuine $\mathrm{CP}$ violation in the neutrino sector. For the golden $v_{\mu} \rightarrow v_{\mathrm{e}}$ channel, the different energy distributions of the two components provide a signature of their separation. At long baselines, they show oscillations in the low and medium energy regions, with zeros at different positions and peculiar behaviour around the zeros. A magic energy $\mathrm{E}=(0.91 \pm 0.01) \mathrm{GeV}$ exists at $\mathrm{L}=1300 \mathrm{~km}$ with vanishing CPT-odd component and maximal genuine $\mathrm{CP}$ asymmetry proportional to $\sin \delta$, with $\delta$ the weak $\mathrm{CP}$ phase. For energies above $1.5 \mathrm{GeV}$, the sign of the $\mathrm{CP}$ asymmetry discriminates the neutrino hierarchy.

In the experimental region of existing and planned terrestrial accelerator neutrinos, it is proved [112] that, at medium baselines -T2K and T2HK-, the CPT-odd component is small and nearly $\delta$-independent, so it can be subtracted from the experimental $\mathrm{CP}$ asymmetry as a theoretical background, provided the hierarchy is known. At long baselines -DUNE-, on the other hand, one finds that (i) a hierarchy-odd term in the CPT-odd component dominates the $\mathrm{CP}$ asymmetry for energies above the first oscillation node, and (ii) the fake CPT-odd term vanishes, independent of the CP phase $\delta$ and the hierarchy, at the magic energy $\mathrm{E}=0.91 \mathrm{GeV}(\mathrm{L} / 1300 \mathrm{~km})$ near the second oscillation maximum, where the genuine T-odd term is almost maximal and proportional to $\sin \delta$. A measurement of the $\mathrm{CP}$ asymmetry in these energy regions would thus provide separate information on (i) the neutrino mass ordering, and (ii) direct evidence of genuine $\mathrm{CP}$ violation in the lepton sector.

\subsection{Global Lepton Number}

Knowing that neutrinos are massive, the most fundamental problem is the determination of the nature of neutrinos with definite mass: are they either four-component Dirac particles with a conserved global lepton number L, distinguishing neutrinos from antineutrinos; or two-component truly neutral (no electric charge and no global lepton number) self-conjugate Majorana particles [98]? For Dirac neutrinos, like quarks and charged leptons, their masses can be generated in the Standard Model of particle physics by spontaneous breaking of the gauge symmetry with the doublet Higgs scalar, if there are additional right-handed sterile neutrinos. However, the Yukawa couplings would then be unnaturally small compared to those of all other fermions. One would also have to explain the origin of the global lepton number avoiding a Majorana mass for these sterile neutrinos. A Majorana $\Delta \mathrm{L}=2$ mass term, with the active left-handed neutrinos only, leads to definite-mass neutrinos with no definite lepton charge. However, there is no way in the Standard Model to generate this Majorana mass, so the important conclusion in fundamental physics arises: Majorana neutrinos would be an irrefutable proof of physics beyond the Standard Model. Due to the Majorana condition of neutrinos with definite mass being their own antiparticles, Majorana neutrinos have two additional CP-violating phases [115-117] beyond the Dirac case.

Neutrino flavour oscillation experiments cannot determine the fundamental nature of massive neutrinos. In order to probe whether neutrinos are Dirac or Majorana particles, the known way has been the search of processes violating the global lepton number L. The difficulty encountered in these studies is well illustrated by the so-called confusion theorem $[118,119]$, stating that in the limit of zero mass, there is no difference between Dirac and Majorana neutrinos. As all known neutrino 
sources produce highly relativistic neutrinos (except for the present cosmic neutrino background in the universe), the $\Delta \mathrm{L}=2$ observables are highly suppressed. $\mathrm{Up}$ to now, there has been a consensus that the highest sensitivity to small Majorana neutrino masses can be reached in experiments on the search of the L-violating neutrinoless double- $\beta$ decay process $(0 v \beta \beta)$. Dozens of experiments around the world are seeking out a positive signal, and the most sensitive limits are obtained by GERDA-II [120] in ${ }^{76} \mathrm{Ge}$, CUORE [121] in ${ }^{130} \mathrm{Te}$ and KAMLAND-Zen [122] in ${ }^{136} \mathrm{Xe}$. An alternative to $0 v \beta \beta$ is provided by the mechanism of neutrinoless double electron capture (0vECEC) [123], which actually corresponds to a virtual mixing between a nominally stable parent $Z$ atom and a daughter $(Z-2)^{*}$ atom with two electron holes. The experimental process is the subsequent $X$-ray emission and it becomes resonantly enhanced when the two mixed atomic states are nearly degenerated. The search of appropriate candidates [124] satisfying the resonant enhancement is being pursued with the precision in the measurement of atomic masses provided by traps. The process can be stimulated [125] in XLaser facilities. The 2vECEC decay, allowed in the Standard Model, has recently been observed for the first time by the XENON Collaboration [126] in ${ }^{124} \mathrm{Xe}$. This last process, contrary to the case of $2 v \beta \beta$ for searches of $0 v \beta \beta$, is not an irreducible background for $0 v E C E C$ when the resonance condition is satisfied.

Recently, a novel idea [127] has been developed following a different path to the search of $\Delta \mathrm{L}=2$ processes. It is based on having a pair of virtual non-relativistic neutrinos of definite mass, whose quantum distinguishability is different for Dirac and Majorana nature due to the lepton charge. Such a physical situation is apparent in the long-range force mediated by two neutrinos at distances near its range of microns, well beyond the atomic scale. Neutral aggregate matter has a weak charge and there is a $3 \times 3$ Hermitian matrix of six coherent charges for its interaction with two neutrinos of equal or different masses. The effective two-neutrino potential near its range leads to observable absolute neutrino mass and the Dirac/Majorana distinction via two effects: a different r-dependence and a violation of the weak equivalence principle.

The search for the elusive neutrino properties of the absolute neutrino mass and the Global Lepton Number symmetry is of primary importance and, although experimentally challenging, is worthwhile to be pursued using all alternative ways.

\section{The Brout-Englert-Higgs Mechanism}

The Standard Model of particle physics contains, as elementary constituents, the three families of fermions with the Quark-Lepton Symmetry. Their interactions appear as a requirement of the local Gauge Symmetries $\mathrm{SU}(3)_{\mathrm{C}} \times \mathrm{SU}(2)_{\mathrm{L}} \times \mathrm{U}(1)_{\mathrm{Y}}$ generated by the three charges of colour, weak isospin and weak hypercharge. The last two combine to the electric charge for $\mathrm{U}(1)_{\mathrm{em}}$. These interactions operate as Exchange Forces with the Mediator Gluons with $\mathrm{m}=0$ but are confined, along with Photons with $\mathrm{m}=0$ and the massive $\mathrm{W}^{ \pm}, \mathrm{Z}$ bosons. The Standard Model not only predicted new particles and interactions, but its agreement with all precision experimental results of detailed Observables in the last few decades is impressive. However, these gauge symmetries are exact only in the massless limit, against the facts in nature. One should have a very subtle mechanism for the Origin of Mass without affecting the interactions, responsible for the $\mathrm{SU}(2)_{\mathrm{L}} \times \mathrm{U}(1)_{\mathrm{Y}}$ gauge symmetry breaking into $\mathrm{U}(1)_{\mathrm{em}}$. This is the Brout-Englert-Higgs Mechanism [128,129].

The Spontaneous ElectroWeak Symmetry Breaking (SEWSB) is based on the possibility that a symmetric Law of Physics can lead to asymmetric solutions. One should be aware that a Quantum Field Theory needs, for its precise definition, not only the Lagrangian (the physical law) but also the Quantum Vacuum, the lowest energy state from which particles are created and annihilated. SEWSB means that the physical law is symmetric, and the vacuum is asymmetric. How?

The spacetime is filled with a "medium", a complex scalar field with the interaction like a "mexican hat" of Figure 10. 


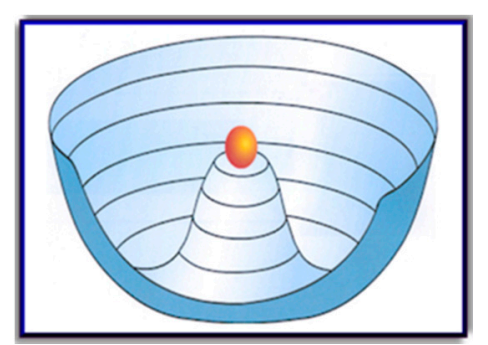

Figure 10. Interaction of the complex scalar field.

This behaviour is obtained from a negative "mass square" quadratic term plus a positive quartic term. We observe that, instead of a unique symmetric lowest energy state, there are many possible vacua and one choice breaks the symmetry. This "spontaneous symmetry breaking" could be called a hidden symmetry because the results are independent of the chosen vacuum.

The physical particle created from the new vacuum is the Higgs Boson, a remnant of the Brout-Englert-Higgs Mechanism, hence its importance. There is a clear signature of the Higgs particle: its coupling to all particles, including to itself, is proportional to their mass, a property that breaks the gauge symmetry. The Origin of Mass comes from the asymmetry of the new vacuum.

On 4 July 2012, the ATLAS and CMS experiments at CERN's Large Hadron Collider announced $[130,131]$ they had each observed a new particle in the mass region around $125 \mathrm{GeV}$. In Figure 11, we show these original data together with the comparison of the measured partial decay rates to different channels to the expected theoretical predictions in the Standard Model.

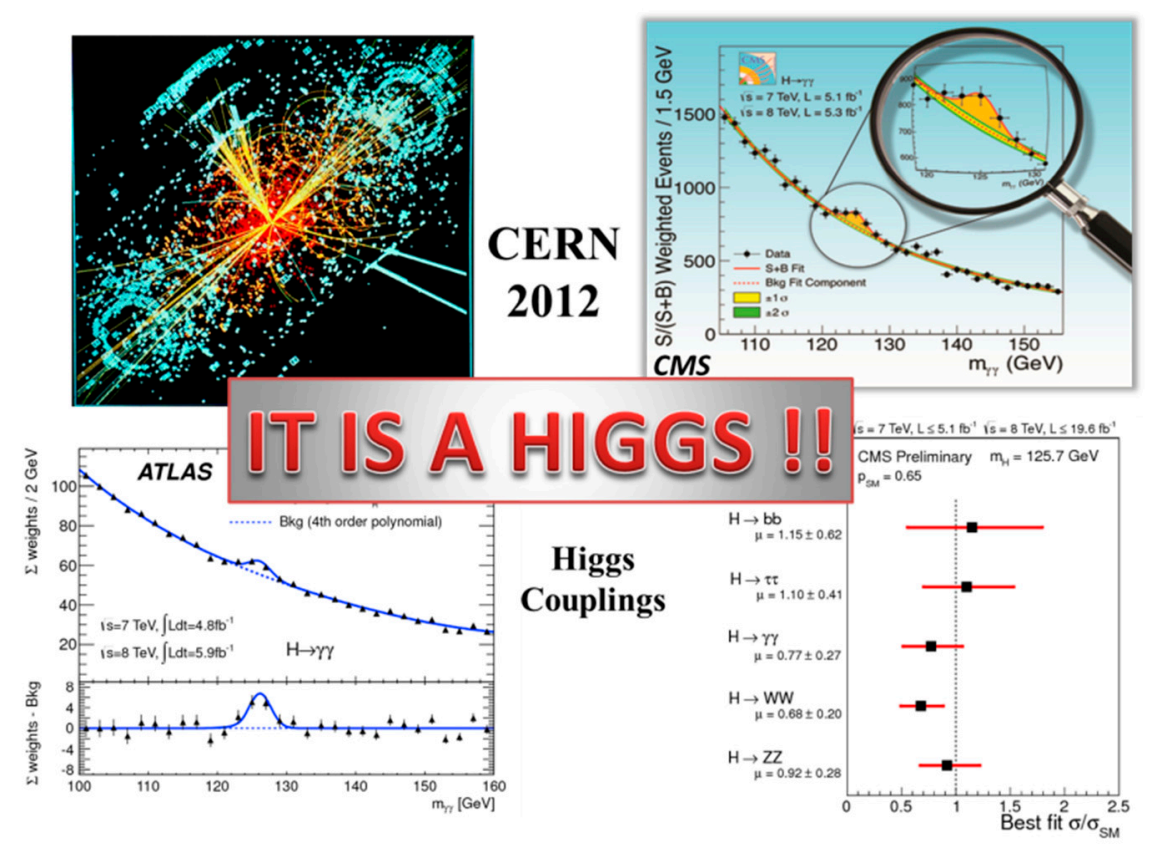

Figure 11. Production and Decay of the Higgs boson observed in the ATLAS and CMS experiments.

As seen, the couplings are consistent with those expected for a Higgs particle.

On 8 October 2013, the Nobel prize in physics was awarded jointly to François Englert and Peter Higgs "for the theoretical discovery of a mechanism that contributes to our understanding of the origin of mass of subatomic particles, and which recently was confirmed through the discovery of the predicted fundamental particle, by the ATLAS and CMS experiments at CERN's Large Hadron Collider." 


\subsection{The Higgs Boson Gauge and Yukawa Couplings}

In 2020, many Higgs properties have already been established, among them, its mass $125 \mathrm{GeV}$, spin/parity $0^{+}$, width $<1 \mathrm{Gev}$ (direct) and $<0.015 \mathrm{Gev}$ (indirect) and the observed direct couplings to Vector Bosons, $\tau$ leptons and top quarks. This is well illustrated in Figure 12 using the combined ATLAS + CMS experimental results.

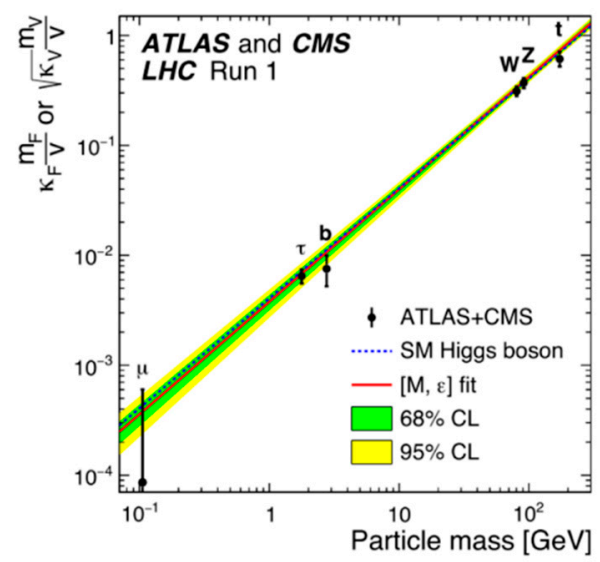

Figure 12. The observed direct couplings of the Higgs to vector bosons $W, Z$ and fermions.

As seen, all measurements are compatible with SM predictions. The decay channel $H \rightarrow b \bar{b}$, the unique final state to measure the coupling with down-type quarks, is the most relevant. It has the largest branching fraction: $58 \%$ for $\mathrm{M}_{\mathrm{H}}=125 \mathrm{GeV}$. The problem, however, is the huge QCD background. Hence the solution of studying the production of $V H(b \bar{b})$ by $q \bar{q}$ with $\mathrm{V}=\mathrm{W}, \mathrm{Z}$. The case $\mathrm{Z} H$ has an additional g-g produced amplitude. The leptonic decay channels of $V$ are preferred $\mathrm{W} \rightarrow 1 \mathrm{v}$ and $Z \rightarrow 11, v v$. ATLAS and CMS have more than $5 \sigma$ separate observations of the $\mathrm{H} \rightarrow \mathrm{b}$ b Decay confirming the SM result on the Yukawa coupling to b's.

The SM Lagrangian, as summarized in Figure 13, contains gauge propagation and self-interaction in the first line, fermion propagation and interaction with gauge fields in the second, the last two lines involving the Higgs Boson. The Yukawa couplings—in red-and the gauge couplings—in green-are present in Higgs Production and in Higgs Decays as advertised by the arrows.

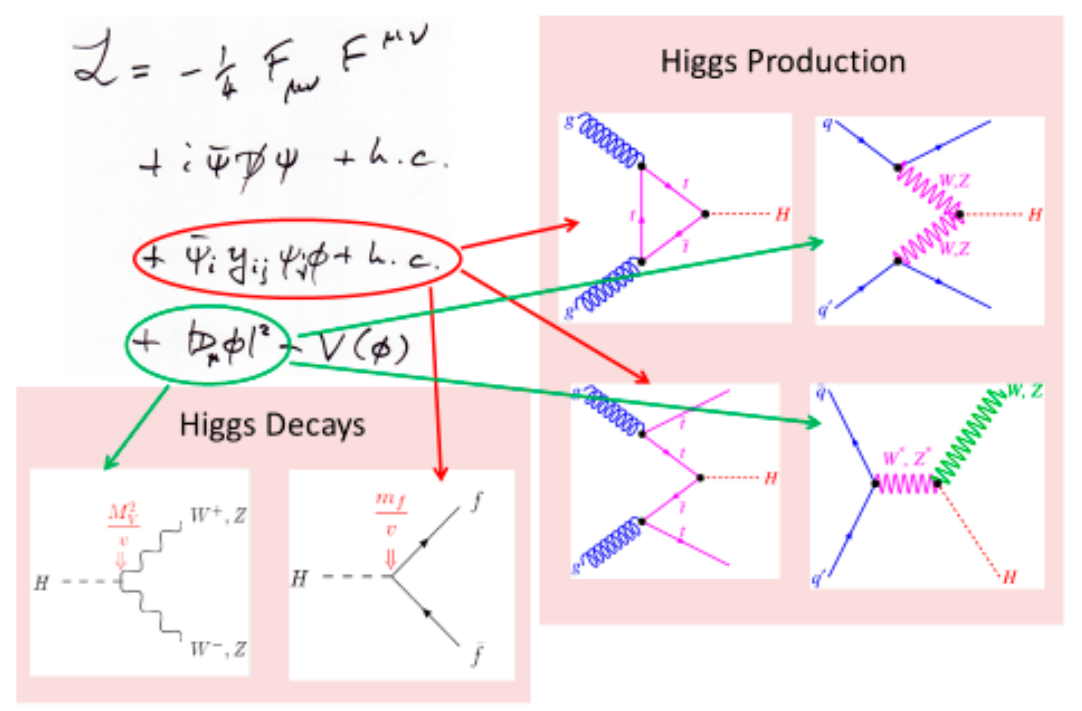

Figure 13. The Higgs Lagrangian and its test by means of Higgs Production and Higgs Decay. 
We may conclude that there is strong evidence that the gauge and Yukawa couplings of the $\mathrm{H}$-particle at $125 \mathrm{GeV}$ correspond to a Higgs. However, the SM Higgs potential $\mathrm{V}(\phi)$ has not been experimentally explored up to now. We need new accessible observables.

\subsection{The Higgs Potential}

The SM scalar sector of the theory is constructed for a doublet under SU(2) of Complex Fields

$$
\phi=\left(\begin{array}{c}
\phi^{+} \\
\phi^{0}
\end{array}\right)
$$

Besides gauge and Yukawa couplings, the HIGGS POTENTIAL is given in Equation (34)

$$
V(\phi)=\mu^{2} \phi^{\dagger} \phi+\lambda\left(\phi^{\dagger} \phi\right)^{2}
$$

and it is invariant under local gauge transformations.

For $\mu^{2}<0, \lambda>0$, the potential energy density $\mathrm{V}(\phi)$ has a minimum at $\phi^{\dagger} \phi=\frac{-\mu^{2}}{2 \lambda}=\frac{\mathrm{v}^{2}}{2}$.

This is a degenerate vacuum, seen in the Mexican hat of Figure 10, as a consequence of gauge invariance. Let us choose the direction $\phi_{0}=\frac{1}{\sqrt{2}}\left(\begin{array}{l}0 \\ \mathrm{v}\end{array}\right), \phi(x)=\frac{1}{\sqrt{2}}\left(\begin{array}{c}0 \\ \mathrm{v}+H(x)\end{array}\right)$, so that $\mathrm{H}(\mathrm{x})$ is the remnant neutral scalar field defined from the new vacuum: the Higgs.

The choice of the direction implies three broken global symmetries leading, therefore, to three massless Goldstone Bosons: two charged and one neutral. Physically, they are associated with the longitudinal degrees of freedom of the massive $\mathrm{W}^{ \pm}$and $\mathrm{Z}$ bosons. When transforming the fields of definite mass, the local gauge symmetry appears broken. This is the "Spontaneous Symmetry Breaking" (SSB) mechanism. The symmetry is still there, hidden in the invariance of the Lagrangian under the transformation of the original non-physical fields, and it is reminded with the independence of physics with the particular choice of the direction responsible for the SSB.

After the SSB, the SM Higgs potential—in terms of the physical Higgs field—becomes

$$
V(H)=\frac{m_{H}^{2}}{2} H^{2}+\lambda_{3}^{S M} \mathbf{v} H^{3}+\lambda_{4}^{S M} H^{4}
$$

where $m_{H}^{2}=-2 \mu^{2}, \lambda_{3}^{S M}=\frac{m_{H}^{2}}{2 \mathrm{v}^{2}}, \lambda_{4}^{S M}=\frac{m_{H}^{2}}{8 \mathrm{v}^{2}}$

The Higgs vacuum expectation value $(\mathrm{VEV}) \mathrm{v}=246 \mathrm{GeV}$ is related to the Fermi coupling constant $\mathrm{G}_{\mathrm{F}}$ measured in muon decay. Thus, we have a self-coupling $\lambda_{3}^{S M}=0.13$.

Equation (35) for the Higgs potential was never probed and it is waiting on its measurement in order to complete the triumph of the SM for explaining the secrets of nature. The self-couplings of the Higgs boson are the keystone.

\section{Conclusions and Outlook}

The three sectors of the Standard Model—Strong, ElectroWeak and Higgs—represent a tribute to the idea that Symmetry, and Symmetry Breaking, is the Guiding Principle for Particles and Interactions and the Origin of Mass. The particle content was induced by Discrete Symmetry Breaking for fermions, opening the field of Flavour Physics for Quarks and Leptons.

We emphasize the role of different definite patterns for the breaking of symmetries, like:

- Mass terms are incompatible with both gauge and chirality symmetries.

- Quantum loop Anomalies break conformal symmetry for vector theories and gauge symmetry for chiral field theories.

- The Particle Content of the theory controls the breaking of Discrete Symmetries CP and T. 
- A gauge Asymmetric Vacuum leads to spontaneous symmetry breaking with hidden gauge symmetry and explaining the Origin of Mass for elementary particles.

What next? There are theoretical and observational reasons for searching Beyond-Standard-Model Physics in LHC experiments and in other facilities. I list some of them:

- Why the quantization of electric charge, Dual QED, Magnetic Monopoles

- The principle of "Threeality" in fundamental physics.

- $\quad$ The Hierarchy Problem for scalars, Supersymmetry.

- $\quad$ Grand Unification, p-decay.

- $\quad$ Neutrino Mass, Mixing, CPV, Global Lepton Number.

- Charged Lepton Flavour Violation.

- Baryon Asymmetry of the Universe, Leptogenesis.

- Dark Matter

- Dark Energy

Most ideas tackling these points are linked to the paradigm that symmetries will continue to be the guiding principle for fundamental physics. Among the Discrete Symmetries, CPT is protected by the "CPT-Theorem" in quantum field theory formulated in Minkowski spacetime with interactions satisfying the Lorentz invariance, locality and unitarity. However, there is nothing at the level of quantum mechanics which forbids having CPT-violation and there are sound quantum-gravity arguments in favour of this ultimate symmetry breaking.

Funding: This research has been supported by the FEDER/MCIyU-AEI Grant FPA2017-84543-P and Generalitat Valenciana Project GVPROMETEO 2017-033.

Acknowledgments: I would like to thank the organizers of this Special Issue for the invitation to present these ideas on the role of symmetries in fundamental physics.

Conflicts of Interest: The author declares no conflict of interest.

\section{References}

1. Gell-Mann, M. A schematic model of baryons and mesons. Phys. Lett. 1964, 8, 214. [CrossRef]

2. Bloom, E.D.; Coward, D.H.; DeStaebler, H.C.; Drees, J.; Miller, G.; Mo, L.W.; Taylor, R.E.; Breidenbach, M.; Friedman, J.I.; Hartmann, G.C.; et al. High-energy inelastic e p scattering at 6 degrees and 10 degrees. Phys. Rev. Lett. 1969, 23, 930. [CrossRef]

3. Hanson, G.; Abrams, G.S.; Boyarski, A.; Breidenbach, M.; Bulos, F.; Chinowsky, W.; Feldman, G.J.; Friedberg, C.E.; Fryberger, D.; Goldhaber, G.; et al. Evidence for jet structure in hadron production by $\mathrm{e}^{+} \mathrm{e}^{-}$annihilation. Phys. Rev. Lett. 1975, 35, 1609. [CrossRef]

4. Brandelik, R.; Braunschweig, W.; Gather, K.; Kadansky, V.; Lubelsmeyer, K.; Mattig, P.; Martyn, H.U.; Peise, G.; Rimkus, J.; Sander, H.G.; et al. Evidence for planar events in $\mathrm{e}^{+} \mathrm{e}^{-}$annihilation at high energies. Phys. Lett. $B$ 1979, 86, 243. [CrossRef]

5. Gross, D.J.; Wilczek, F. Ultraviolet behavior of nonabelian gauge theories. Phys. Rev. Lett. 1973, 30, 1343. [CrossRef]

6. Politzer, H.D. Reliable perturbative results for strong interactions? Phys. Rev. Lett. 1973, 30, 1346. [CrossRef]

7. Lee, T.D.; Yang, C.N. Question of parity conservation in weak interactions. Phys. Rev. 1956, 104, 254. [CrossRef]

8. Glashow, S.L. Partial symmetries of weak interactions. Nucl. Phys. 1961, 22, 579. [CrossRef]

9. Weinberg, S. A model of leptons. Phys. Rev. Lett. 1967, 19, 1264. [CrossRef]

10. Salam, A. Weak and electromagnetic interactions. Conf. Proc. C 1968, 680519, 367.

11. Hasert, F.J.; Kabe, S.; Krenz, W.; von Krogh, J.; Lanske, D.; Morfin, J.; Schultze, K.; Weerts, H.; Bertrand-Coremans, G.H.; Sacton, J.; et al. Observation of neutrino-like interactions without muon on electron in the gargamelle neutrino experiment. Phys. Lett. B 1973, 46, 138. [CrossRef] 
12. Arnison, G.; Astbury, A.; Aubert, B.; Bacci, C.; Bauer, G.; Bézaguet, A.; Böck, R.; Bowcock, T.J.V.; Calvetti, M.; Catz, P.; et al. Experimental observation of lepton pairs of invariant mass around $95 \mathrm{GeV} / \mathrm{c}^{2}$ at the CERN SPS collider. Phys. Lett. B 1983, 126, 398. [CrossRef]

13. Bagnaia, P.; Banner, M.; Battiston, R.; Bloch, P.; Bonaudi, F.; Borer, K.; Borghini, M.; Chollet, J.C.; Clark, A.G.; Conta, C.; et al. Evidence for $\mathrm{Z}^{0} \rightarrow \mathrm{e}^{+} \mathrm{e}^{-}$at the CERN $\bar{p} p$ collider. Phys. Lett. B 1983, 129, 130. [CrossRef]

14. Cabibbo, N. Unitary symmetry and leptonic decays. Phys.Rev.Lett. 1963, 10, 531. [CrossRef]

15. Glashow, S.L.; Iliopoulos, J.; Maiani, L. Weak interactions with lepton-hadron symmetry. Phys. Rev. D 1970, 2, 1285. [CrossRef]

16. Aubert, J.J.; Becker, U.; Biggs, P.J.; Burger, J.; Chen, M.; Everhart, G.; Goldhagen, P.; Leong, J.; McCorriston, T.; Rhoades, T.G.; et al. Experimental observation of a heavy particle J. Phys. Rev. Lett. 1974, 33, 1404. [CrossRef]

17. Augustin, J.E.; Boyarski, A.; Breidenbach, M.; Bulos, F.; Dakin, J.T.; Feldman, G.J.; Fischer, G.E.; Fryberger, D.; Hanson, G.; Jean-Marie, B.; et al. Discovery of a narrow resonance in $\mathrm{e}^{+} \mathrm{e}^{-}$annihilation. Phys. Rev. Lett. 1974, 33, 1406. [CrossRef]

18. Kobayashi, M.; Maskawa, T. CP violation in the renormalizable theory of weak interactions. Prog. Theor. Phys. 1973, 49, 652. [CrossRef]

19. Albajar, C. Search for $B^{0}-\bar{B}^{0}$ oscillations at the CERN $p-\bar{p}$ collider. Phys. Lett. $B$ 1987, 186, 247, Erratum in Phys. Lett. B 1987, 197, 565. [CrossRef]

20. Albrecht, H.; Andam, A.A.; Binder, U.; Bockmann, P.; Glaser, R.; Harder, G.; Nippe, A.; Schafer, M.; Schmidt-Parzefall, W.; Schroder, H.; et al. Observation of $B^{0}-\bar{B}^{0}$ mixing. Phys. Lett. B 1987, $192,245$. [CrossRef]

21. Veltman, M.J.G. Radiative corrections to vector boson masses. Phys. Lett. B 1980, 91, 95. [CrossRef]

22. Bernabeu, J.; Pich, A.; Santamaría, A. $\Gamma(\mathrm{Z} \rightarrow b-\bar{b})$ : A signature of hard mass terms for a heavy top. Phys. Lett. $B$ 1988, 200, 569. [CrossRef]

23. Abe, F.; Akimoto, H.; Akopian, A.; Albrow, M.G.; Amendolia, S.R.; Amidei, D.; Antos, J.; Anway-Wiese, C.; Aota, S.; Apollinari, G.; et al. Observation of top quark production in $\bar{p} p$ collisions. Phys. Rev. Lett. 1995, 74, 2626. [CrossRef] [PubMed]

24. Abachi, S.; Abbott, B.; Abolins, M.; Acharya, B.S.; Adam, I.; Adams, D.L.; Adams, M.; Ahn, S.; Aihara, H.; Alitti, J.; et al. Observation of the top quark. Phys. Rev. Lett. 1995, 74, 2632. [CrossRef] [PubMed]

25. Fujikawa, K.; Suzuki, H. Path Integrals and Quantum Anomalies; Clarendon Press: Oxford, UK, 2004.

26. Christenson, J.H.; Cronin, J.W.; Fitch, V.L.; Turlay, R. Evidence for the $2 \pi$ Decay of the $K_{2}^{0}$ Meson. Phys. Rev. Lett. 1964, 13, 138. [CrossRef]

27. NA31 Collaboration. First evidence for direct CP violation. Phys. Lett. B 1988, 206, 169. [CrossRef]

28. NA48 Collaboration. A new measurement of direct $\mathrm{CP}$ violation in two pion decays of the neutral kaon. Phys. Lett. B 1999, 465, 335. [CrossRef]

29. CPLEAR Collaboration. Physics at CPLEAR. Phys. Rep. 2003, 374, 165. [CrossRef]

30. BABAR Collaboration. Observation of CP violation in the B0 meson system. Phys. Rev. Lett. 2001, 87, 091801. [CrossRef]

31. BELLE Collaboration. Observation of large CP violation in the neutral B meson system. Phys. Rev. Lett. 2001, 87, 091802. [CrossRef]

32. LHCb Collaboration. Observation of CP violation in charm decays. Phys. Rev. Lett. 2019, 122, 211803. [CrossRef] [PubMed]

33. Banuls, M.C.; Bernabéu, J. CP, T and CPT versus temporal asymmetries for entangled states of the $\mathrm{B}(\mathrm{d})$ system. Phys. Lett. B 1999, 464, 117-122. [CrossRef]

34. Lees, J.P.; Poireau, V.; Tisserand, V.; Tico, J.G.; Grauges, E.; Palanoab, A.; Eigen, G.; Stugu, B.; Brown, D.N.; Kerth, L.T.; et al. Observation of time reversal violation in the $\mathrm{B}^{0}$ meson system. Phys. Rev. Lett. 2012, 109, 211801. [CrossRef] [PubMed]

35. Bernabeu, J.; Martinez-Vidal, F.; Villanueva-Perez, P. Time reversal violation from the entangled B ${ }^{0}$-antiB ${ }^{0}$ system. J. High Energy Phys. 2012, 2012, 64. [CrossRef]

36. Bernabeu, J.; Botella, F.J.; Nebot, M. Genuine T, CP, CPT asymmetry parameters for the entangled B(d) system. J. High Energy Phys. 2016, 2016, 100. [CrossRef]

37. Bernabeu, J.; Di Domenico, A.; Villanueva-Perez, P. Direct test of Time Reversal symmetry in the entangled neutral Kaon system at a $\Phi$-Factory. Nucl. Phys. B 2013, 868, 102. [CrossRef] 
38. CPLEAR Collaboration. A determination of the CPT violation parameter $\operatorname{Re}(\delta)$ from the semileptonic decay of strangeness tagged neutral Kaons. Phys. Lett. B 1998, 444, 52. [CrossRef]

39. KTeV Collaboration. A measurement of the $\mathrm{K}_{\mathrm{L}}$ charge asymmetry. Phys. Rev. Lett. 2002, 88, 181601. [CrossRef]

40. KLOE Collaboration. Study of the branching ratio and charge asymmetry for the decay $\mathrm{K}_{\mathrm{S}} \rightarrow \pi \mathrm{e} v$ with the KLOE detector. Phys. Lett. B 2006, 636, 173. [CrossRef]

41. Bernabeu, J.; Di Domenico, A.; Villanueva-Perez, P. Probing CPT in transitions with entangled neutral Kaons. JHEP 2015, 1510, 139. [CrossRef]

42. Bernabeu, J.; Mavromatos, N.; Papavassiliou, J. Novel type of CPT Violation for correlated EPR states. Phys. Rev. Lett. 2004, 92, 131601. [CrossRef] [PubMed]

43. Bernabeu, J.; Mavromatos, N.; Sarkar, S. Decoherence induced CPT Violation and entangled neutral mesons. Phys. Rev. D 2006, 74, 045014. [CrossRef]

44. ALPHA Collaboration. Characterization of the 1S-2S transition in antihydrogen. Nature 2018, 557, 71. [CrossRef] [PubMed]

45. ALPHA Collaboration. Observation of the hyperfine spectrum of antihydrogen. Nature 2017, 548,66 . [CrossRef]

46. ASACUSA Collaboration. A hydrogen beam to characterize the ASACUSA antihydrogen hyperfine spectrometer. Nucl. Instrum. Meth. A 2019, 935, 110. [CrossRef]

47. Super-Kamiokande Collaboration. Evidence for oscillation of atmospheric neutrinos. Phys. Rev. Lett. 1998, 81, 1562. [CrossRef]

48. SNO Collaboration. Direct Evidence for neutrino flavor transformation from neutral current interactions in the Sudbury Neutrino Observatory. Phys. Rev. Lett. 2002, 89, 011301. [CrossRef]

49. KamLAND Collaboration. First results from KamLAND: Evidence for reactor anti-neutrino disappearance. Phys. Rev. Lett. 2003, 90, 021802. [CrossRef]

50. K2K Collaboration. Measurement of neutrino oscillation by the K2K experiment. Phys. Rev. D 2006, 74, 072003. [CrossRef]

51. Pontecorvo, B. Mesonium and anti-mesonium. Sov. Phys. JETP 1957, 6, 429.

52. Maki, Z.; Nakagawa, M.; Sakata, S. Remarks on the unified model of elementary particles. Prog. Theor. Phys. 1962, 28, 870. [CrossRef]

53. Capozzi, F.; Fogli, G.L.; Lisi, E.; Marrone, A.; Montanino, D.; Palazzo, A. Status of three-neutrino oscillation parameters, circa 2013. Phys. Rev. D 2014, 89, 093018. [CrossRef]

54. Esteban, I.; Gonzalez-Garcia, M.C.; Maltoni, M.; Martinez-Soler, I.; Schwetz, T. Updated Fit to three neutrino mixing: Exploring accelerator-reactor complementarity. J. High Energy Phys. 2017, 2017, 87. [CrossRef]

55. De Salas, P.F.; Forero, D.V.; Ternes, C.A.; Tortola, M.; Valle, J.W.F. Status of neutrino oscillation 2018: $3 \sigma$ hint for normal mass ordering and improved CP sensitivity. Phys. Lett. B 2018, 782, 633. [CrossRef]

56. Hyper-Kamiokande Collaboration. Hyper-Kamiokande design report. arXiv 2018, arXiv:1805.04163. [physics.ins-det].

57. DUNE Collaboration. Long Baseline Neutrino Facility (LBNF) and Deep Underground Neutrino Experiment (DUNE): Conceptual design report, Volume 2: The physics program for DUNE at LBNF. arXiv 2015, arXiv:1512.06148. [physics.ins-det].

58. T2K Collaboration. Indication of electron neutrino appearance from an accelerator-produced off-axis muon neutrino beam. Phys. Rev. Lett. 2011, 107, 041801. [CrossRef]

59. Double Chooz Collaboration. Indication of reactor antinue disappearance in the double chooz experiment. Phys. Rev. Lett. 2012, 108, 131801. [CrossRef]

60. Daya Bay Collaboration. Observation of electron-antineutrino disappearance at Daya Bay. Phys. Rev. Lett. 2012, 108, 171803. [CrossRef]

61. RENO Collaboration. Observation of electron-antineutrino diappearance at RENO. Phys. Rev. Lett. 2012, 108, 191802. [CrossRef]

62. Fukugita, M.; Yanagida, T. Baryogenesis without grand unification. Phys. Lett. B 1986, 174, 45. [CrossRef]

63. Thomson, J.J. XL. Cathode rays. Lond. Edinb. Dublin Philos. Mag. J. Sci. 1897, 44, 293-316. [CrossRef]

64. Rutherford, E. LIV. Collision of a particles with light atoms. IV. An anomalous effect in nitrogen. Lond. Edinb. Dublin Philos. Mag. J. Sci. 1919, 37, 581-587. [CrossRef] 
65. Rutherford, E. LXXIX. The scattering of $\alpha$ and $\beta$ particles by matter and the structure of the atom. Lond. Edinb. Dublin Philos. Mag. J. Sci. 1911, 21, 669-688.

66. Rutherford, E. VIII. Uranium radiation and the electrical conduction produced by it. Lond. Edinb. Dublin Philos. Mag. J. Sci. 1899, 47, 109-163. [CrossRef]

67. Becquerel, H. Influence d'un champ magnétique sur le rayonnement des corps radio-actifs. J. Phys. Théorique Appliquée 1900, 9, 71-78. [CrossRef]

68. Von Baeyer, O.; Hahn, O.; Meitner, L. Uber die $\beta$-Strahlen des aktiven niederschlags des thoriums. Phys. Z. 1911, 12, 273.

69. Danysz, J.; Duane, W. Sur les charges électriques des rayons $\alpha$ et $\beta$. Le Radium 1912, 9, 417-421. [CrossRef]

70. Chadwick, J. Intensitätsverteilung im magnetischen Spectrum der $\beta$-Strahlen von radium B + C. Verhandl. Dtsc. Phys. Ges. 1914, 16, 383.

71. Ornstein, L.; Van Wijk, W.Z. Untersuchungen über das negative Stickstoff bandenspektrum. Für Phys. 1928, 49, 315-322. [CrossRef]

72. Chadwick, J.; Ellis, C.D. A preliminary investigation of the intensity distribution in the $\beta$-ray spectra of radium B and C. In Proceedings of the Cambridge Philosophical Society; Cambridge University Press: Cambridge, UK, 1922; Volume 21, p. 274.

73. Ellis, C.D.; Wooster, W. The $\beta$-ray Type of Disintegration. In Mathematical Proceedings of the Cambridge Philosophical SocietyI; Cambridge University Press: Cambridge, UK, 1925; Volume 22, p. 849.

74. Ellis, C.D.; Wooster, W.A. The average energy of disintegration of radium E. Proceedings of the Royal Society of London. Series A. Contain. Pap. Math. Phys. Character 1927, 117, 109-123.

75. Bohr, N.R. Atomic stability and conservation laws. Accad. d'Italia 1932, 119-130.

76. Chadwick, J. Possible existence of a neutrón. Nature 1932, 129, 312. [CrossRef]

77. Anderson, C.D. The Positive electron. Phys. Rev. 1933, 43, 491. [CrossRef]

78. Fermi, E. Tentativo di una teoria dei raggi $\beta$. Nuovo Cim. 1934, 11, 1-19. [CrossRef]

79. Bethe, H.; Peierls, R. The 'neutrino'. Nature 1934, 133, 532. [CrossRef]

80. Cowan, C.L.; Reines, F.; Harrison, F.B.; Kruse, H.W.; McGuire, A.D. Detection of the free neutrino: A Confirmation. Science 1956, 124, 103. [CrossRef]

81. Anderson, C.D.; Neddermeyer, S.H. Cloud chamber observations of cosmic rays at 4300 meters elevation and near sea-level. Phys. Rev. 1936, 50, 263. [CrossRef]

82. Lattes, C.M.G.; Muirhead, H.; Occhialini, G.P.S.; Powell, C.F. Processes involving charged mesons. Nature 1947, 159, 694. [CrossRef]

83. Feynman, R.P.; Gell-Mann, M. Theory of Fermi interaction. Phys. Rev. 1958, 109, 193. [CrossRef]

84. Pontecorvo, B. Nuclear capture of mesons and the meson decay. Phys. Rev. 1947, 72, 246. [CrossRef]

85. Pontecorvo, B. Electron and Muon Neutrinos. J. Phys. 1959, 43, 8-221.

86. Pontecorvo, B. Electron and Muon Neutrinos. Sov. Phys. JETP 1960, 10, 1236.

87. Feinberg, G. Decays of the mu meson in the intermediate-meson theory. Phys. Rev. 1958, 110, 1482. [CrossRef]

88. Bartlett, D.; Devons, S.; Sachs, A.M. Search for the decay mode: $\mu \rightarrow \mathrm{e}+\gamma$. Phys. Rev. Lett. 1962, 8, 120. [CrossRef]

89. Danby, G.; Gaillard, J.M.; Goulianos, K.A.; Lederman, L.M.; Mistry, N.B.; Schwartz, M.; Steinberger, J. Observation of high-energy neutrino reactions and the existence of two kinds of neutrinos. Phys. Rev. Lett. 1962, 9, 36. [CrossRef]

90. Perl, M.L.; Abrams, G.S.; Boyarski, A.; Breidenbach, M.; Briggs, D.; Bulos, F.; Chinowsky, W.; Dakin, J.T.; Feldman, G.J.; Friedberg, C.E.; et al. Evidence for anomalous lepton production in e+-e- annihilation. Phys. Rev. Lett. 1975, 35, 1489. [CrossRef]

91. DONUT Collaboration. Observation of $\tau$ neutrino interactions. Phys. Lett. B 2001, 504, 218. [CrossRef]

92. ALEPH, DELPHI, L3, OPAL, SLD, LEP Electroweak Working Group; SLD Electroweak Group; SLD Heavy Flavour Group Collaboration. Precision electroweak measurements on the Z resonance. Phys. Rept. 2006, 427, 257. [CrossRef]

93. Weyl, H. Electron and gravitation. 1. Z. Phys. 1929, 56, 330. (In German) [CrossRef]

94. Wu, C.S.; Ambler, E.; Hayward, R.W.; Hoppes, D.D.; Hudson, R.P. Experimental test of parity conservation in beta decay. Phys. Rev. 1957, 105, 1413. [CrossRef]

95. Goldhaber, M.; Grodzins, L.; Sunyar, A.W. Helicity of neutrinos. Phys. Rev. 1958, 109, 1015. [CrossRef]

96. Pontecorvo, B. Chalk river report. Rep. PD 1946, 205, 174. 
97. Davis, R., Jr.; Harmer, D.S. Attempt to observe the $\mathrm{Cl}^{37}\left(\bar{v} e^{-}\right) \mathrm{Ar}^{37}$ reaction induced by reactor antineutrinos. Bull. Am. Phys. Soc. 1959, 4, 217.

98. Majorana, E. Teoria simmetrica dell'elettrone e del positrone. Nuovo Cim. 1937, 14, 171. [CrossRef]

99. Pontecorvo, B. Inverse beta processes and nonconservation of lepton charge. Sov. Phys. JETP 1958, 7, 172.

100. KamLAND Collaboration Measurement of neutrino oscillation with KamLAND: Evidence for spectral distortion. Phys. Rev. Lett. 2005, 94, 081801. [CrossRef]

101. Pontecorvo, B. Neutrino experiments and the problem of conservation of leptonic charge. Sov. Phys. JETP 1968, 26, 984 .

102. Davis, R., Jr.; Harmer, D.S.; Hoffman, K.C. Search for neutrinos from the sun. Phys. Rev. Lett. 1968, $20,1205$. [CrossRef]

103. Kamiokande-II Collaboration. Experimental study of the atmospheric neutrino flux. Phys. Lett. B 1988, 205, 416. [CrossRef]

104. Bernabeu, J. On the history of the PMNS Matrix... with today's perspective. Nuovo Cim. C037 2014, 3, 145.

105. T2K Collaboration. Constraint on the Matter-Antimatter Symmetry Violating Phase in neutrino oscillations. Nat. 580 2020, 7803, 39.

106. Wolfenstein, L. Neutrino oscillations in matter. Phys. Rev. D 1978, 17, 2369. [CrossRef]

107. Mikheyev, S.P.; Smirnov, A.Y. Resonance amplification of oscillations in matter and spectroscopy of solar neutrinos. Sov. J. Nucl. Phys. 1985, 42, 913.

108. Donini, A.; Gavela, M.B.; Hernandez, P.; Rigolin, S. Neutrino mixing and CP violation. Nucl. Phys. B 2000, 574, 23. [CrossRef]

109. Akhmedov, E.K.; Maltoni, M.; Smirnov, A.Y. Neutrino oscillograms of the Earth: Effects of 1-2 mixing and CP Violation. J. High Energy Phys. 2008, 2008, 072. [CrossRef]

110. Ohlsson, T.; Zhang, H.; Zhou, S. Probing the leptonic Dirac CP-violating phase in neutrino oscillation experiments. Phys. Rev. D 2013, 87, 53006. [CrossRef]

111. Bernabeu, J.; Segarra, A. Disentangling genuine from matter-induced CP Violation in neutrino oscillations. Phys. Rev. Lett. 2018, 121, 211802. [CrossRef]

112. Bernabeu, J.; Segarra, A. Signatures of the genuine and matter-induced components of the CP violation asymmetry in neutrino oscillations. J. High Energy Phys. 2018, 2018, 63. [CrossRef]

113. Bernabeu, J.; Banuls, M.C. CP and T violation in neutrino oscillations. Nucl. Phys. Proc. Suppl. 2000, 87, 315. [CrossRef]

114. Bernabeu, J.; Segarra, A. Do T-asymmetries for neutrino oscillations in uniform matter have a CP-even component? J. High Energy Phys. 2019, 2019, 103. [CrossRef]

115. Bilenky, S.M.; Hosek, J.; Petcov, S.T. On oscillations of neutrinos with dirac and majorana masses. Phys. Lett. B 1980, 94B, 495. [CrossRef]

116. Doi, M.; Kotani, T.; Nishiura, H.; Okuda, K.; Takasugi, E. CP violation in majorana neutrinos. Phys. Lett. B 1981, 102B, 323. [CrossRef]

117. Bernabeu, J.; Pascual, P. CP properties of the leptonic sector for majorana neutrinos. Nucl. Phys. B 1983, $228,21$. [CrossRef]

118. Case, K.M. Reformulation of the majorana theory of the neutrino. Phys. Rev. 1957, 107, 307. [CrossRef]

119. Ryan, C.; Okubo, S. On the equivalence of the Majorana and two-component theories of the neutrino. Nuovo Cim. Suppl. 1964, 2, 234.

120. GERDA Collaboration. Improved limit on neutrinoless double- $\beta$ decay of ${ }^{76}$ Ge from GERDA phase II. Phys. Rev. Lett. 2018, 120, 132503. [CrossRef]

121. CUORE Collaboration. improved limit on neutrinoless double- $\beta$ decay in ${ }^{130} \mathrm{Te}$ with CUORE. Phys. Rev. Lett. 2020, 124, 122501. [CrossRef]

122. KamLAND-Zen. Search for majorana neutrinos near the inverted mass hierarchy region with kamland-zen. Phys. Rev. Lett. 2016, 117, 082503, Addendum in Phys. Rev. Lett. 2016, 117, 109903. [CrossRef]

123. Bernabeu, J.; De Rujula, A.; Jarlskog, C. Neutrinoless double electron capture as a tool to measure the $v_{\mathrm{e}}$ mass. Nucl. Phys. B 1983, 223, 15.

124. Eliseev, S.; Roux, C.; Blaum, K.; Block, M.; Droese, C.; Herfurth, F.; Kluge, H.J.; Krivoruchenko, M.I.; Novikov, Y.N.; Minaya Ramirez, E.; et al. Resonant enhancement of neutrinoless double-electron capture in Gd-152. Phys. Rev. Lett. 2011, 106, 052504. [CrossRef] [PubMed] 
125. Bernabeu, J.; Segarra, A. Stimulated transitions in resonant atom Majorana mixing. J. High Energy Phys. 2018, 2018, 17. [CrossRef]

126. XENON Collaboration. Observation of two-neutrino double electron capture in ${ }^{124}$ Xe with XENON1T. Nature 2019, 568, 532. [CrossRef] [PubMed]

127. Segarra, A.; Bernabeu, J. Absolute neutrino mass and the Dirac/Majorana distinction from the weak interaction of aggregate matter. Phys. Rev. D 2020, 101, 093004. [CrossRef]

128. Englert, F.; Brout, R. Broken symmetry and the mass of gauge vector mesons. Phys. Rev. Lett. 1964, $13,321$. [CrossRef]

129. Higgs, P.W. Broken symmetries and the mass of gauge bosons. Phys. Rev. Lett. 1964, 13, 508. [CrossRef]

130. Aad, G.; Abajyan, T.; Abbott, B.; Abdallah, J.; Khalek, S.A.; Abdelalim, A.A.; Abdinov, O.; Aben, R.; Abi, B.; Abolins, M.; et al. Observation of a new particle in the search for the Standard Model Higgs boson with the ATLAS detector at the LHC. Phys. Lett. B 2012, 716, 1. [CrossRef]

131. Chatrchyan, S.; Khachatryan, V.; Sirunyan, A.M.; Tumasyan, A.; Adam, W.; Aguilo, E.; Bergauer, T.; Dragicevic, M.; Erö, J.; Fabjan, C.; et al. Observation of a new boson at a mass of $125 \mathrm{GeV}$ with the CMS experiment at the LHC. Phys. Lett. B 2012, 716, 30. [CrossRef]

(C) 2020 by the author. Licensee MDPI, Basel, Switzerland. This article is an open access article distributed under the terms and conditions of the Creative Commons Attribution (CC BY) license (http://creativecommons.org/licenses/by/4.0/). 